\title{
Coherent neutrino radiation in supernovae at two loops
}

\author{
A. Sedrakian and A. E. L. Dieperink \\ Kernfysisch Versneller Instituut, NL-9747 AA Groningen, The Netherlands
}

\begin{abstract}
We develop a neutrino transport theory, in terms of the real-time nonequilibrium Green's functions, which is applicable to physical conditions arbitrary far from thermal equilibrium. We compute the coherent neutrino radiation in cores of supernovae by evaluating the two-particle-two-hole $(2 \mathrm{p}$ $2 \mathrm{~h}$ ) polarization function with dressed propagators. The propagator dressing is carried out in the particle-particle channel to all orders in the interaction. We show that at two loops there are two distinct sources of coherence effects in the bremsstrahlung. One is the generically off-shell intermediate state propagation, which leads to the Landau-Pomeranchuk-Migdal type suppression of radiation. We extend previous perturbative results, obtained in the leading order in quasiparticle width, by deriving the exact non-perturbative expression. A new contribution due to off-shell finial/initial baryon states is treated in the leading order in the quasiparticle width. The latter contribution corresponds to processes of higher order than second order in the virial expansion in the number of quasiparticles. At $2 \mathrm{p}-2 \mathrm{~h}$ level, the time component of the polarization tensor for the vector transitions vanishes identically in the soft neutrino approximation. Vector current thereby is conserved. The contraction of the neutral axial vector current with tensor interaction among the baryons leads to a non-vanishing contribution to the bremsstrahlung rate. These rates are evaluated numerically for finite temperature pure neutron matter at and above the nuclear saturation density.
\end{abstract}




\section{INTRODUCTION}

Neutrino production in baryon encounters is among the fundamental processes by which compact stars lose their energy. The reactions can be arranged, in general, according to the number of participating baryons, as the phase space arguments play the central role in controlling their temperature and density dependence [1-6]. In the case of neutrino pair bremsstrahlung, the leading order process in the density virial expansion is the two-body reaction

$$
B_{1}+B_{2} \rightarrow B_{1}+B_{2}+\nu_{f}+\bar{\nu}_{f}
$$

where $B$ stands for a baryon, $\nu_{f}\left(\bar{\nu}_{f}\right)$ for a neutrino (anti-neutrino) of flavor $f=e, \mu, \tau$. Note that the subleading order process (i.e. the one in the absence of the spectator) vanishes for identical particles, as an on-shell propagating particle cannot radiate.

The matter in neutron stars is highly degenerate for temperatures typically below a $\mathrm{MeV}$ and the elementary excitations are quasiparticles with well-defined energy-momentum relation. Produced neutrinos are typically "soft" with energies of order of temperature. In this limit the intermediate quasiparticle propagator diverges as $1 / \omega$ and the amplitudes of the neutrino absorption, scattering, and emission turn out formally divergent as $1 / \omega^{2}$. The infrared behaviour of the in-medium rates, however, is dominated by the neutrino phase space factors, rather than the infrared divergence of the amplitudes and the rates of the bremsstrahlung and its space-like analogues remain finite. At the same time, at low temperatures, the contribution from the infrared region to the rate of the bremsstrahlung is negligible. The combined effect of the cancellation of the infrared divergence and the vanishing contribution from the low frequency region makes the quasiparticle approximation to eq. (1) applicable in cold neutron stars.

During the first several tens of seconds after a supernova explosion and core collapse the temperature of the dense nuclear matter is of the order of several tens of $\mathrm{MeV}$. The neutrino bremsstrahlung is then suppressed, because the formation length of the neutrino radiation is of the same order of magnitude as the mean free path of a baryon $[7-10$. The collective effects become important on the radiation scale (i.e. the role of the spectator in the reaction (11) is taken over by the medium) because the baryon undergoes multiple scattering during the radiation. The underlying mechanism is the Landau-Pomeranchuk-Migdal (LPM) quenching of the radiation, first introduced in the context of QED [11]. The central role in the theory is played by the formation length of radiation $l_{\mathrm{f}}$. If the mean-free-path of a baryon is much larger that the formation length $l_{\mathrm{mfp}} \gg l_{\mathrm{f}}$ then the radiation reduces to a sum of separate radiation events, each of which is well described by the Bethe-Heitler spectrum. In the opposite limit $l_{\mathrm{mfp}} \ll l_{\mathrm{f}}$ the individual scattering events are unresolved and the radiation spectrum takes the Bethe-Heitler form for a single scattering event. In the intermediate regime, when $l_{\mathrm{mfp}} \sim l_{\mathrm{f}}$, the radiation amplitudes for scattering off various centers interfere destructively and the radiation is suppressed (Landau-Pomeranchuk-Migdal effect; for a review see refs. [9, 12]).

The rates of neutrino-nucleon processes are commonly expressed through phase space

integrals over the contraction of the weak currents with the polarization function of the nuclear medium. The polarization function (or structure function) of the supernova/neutron star matter has been subject of many studies [9, 13, 18]. The modifications of reactions rates 
by the spatial correlations among (on-shell) quasiparticles have been studied within the Fermi-liquid theory [13], the one-boson exchange interaction theory [14], the relativistic random phase approximation [15], the variational approach [16], and combinations thereof [17,18]. The spatial correlations tend to suppress reaction rates in general, although their impact on the supernova physics is model dependent [13 18].

The common strategy of incorporating the LPM effect in the neutrino-nucleon interaction processes is to add a quasiparticle damping in the intermediate state propagator by replacing $\omega$ by $\omega+i \gamma[7.9]$. In the soft neutrino limit the vector current coupling does not contribute by virtue of the vector current conservation (CVC) and the net contribution comes from the axial-vector transitions via baryon spin-flip. The above modification of the intermediate state propagator then leads to an ansatz for the nucleon spin structure function: $S_{\sigma} \propto$ $\gamma_{\sigma} /\left(\omega^{2}+\gamma_{\sigma}^{2}\right)$ [] $[9]$, where $\gamma_{\sigma}$ is the nucleon spin-flip collision rate. The ansatz generalizes the quasiparticle picture, in a semi-phenomenological manner, by including the temporal correlations among the quasiparticles in the leading order in the quasiparticle width. The microscopic justification of this phenomenology emerges from the various formulations of the finite temperature quantum filed theory, e. g. the thermo-field dynamics [19] or the closed diagram formalism in the Schwinger-Keldysh technique [12]. A microscopic computation is not straightforward, however. For example, the polarization function of the medium can be computed at one-loop, including the quasiparticle width to all orders in $\gamma$, however a priori the current conservation is not guaranteed at this level. The reason, in part, is that the "more complicated" higher order in loop expansion diagrams contribute at the same order as the single loop [12].

In a previous paper we carried out a microscopic computation of the bremsstrahlung, including the LPM effect, at the one-loop level in a formalism based on the quasiclassical Kadanoff-Baym transport equation [20]. Here we extend this computation to two-loops and partially modify our approach to include the propagator and vertex renormalization on the same footing and to including the tensor force explicitly. The extension to two-loops is motivated by the following. The long range phenomena, driven by the weaker attractive part of the baryon-baryon interaction, are sensitive to the resummation in the particle-hole $(p h)$ channel. On the other hand, as well known, one should fully resum the particleparticle $(p p)$ channel to treat the hard core of the baryon-baryon interaction. Therefore, the $p h$ channel can be treated perturbatively by expanding in the number of particle-hole loops, while the $p p$ channel must be treated non-perturbatively by a full resummation of the ladder diagrams. Thus, the separation of the long range and short range phenomena dictates the manner in which the diagrammatic expansion is carried out. The dressing of the single particle propagators occurs in both channels and can be treated either explicitly, say, by considering higher order self-energies attached to a propagator, or, alternatively, by condensing it in the width of the propagator spectral function. As a consequence of the separation of the scales, the short-range correlations can be condensed in the propagator width on the scales relevant for the long-range phenomena. The imaginary part of a single loop in the $p h$ channel vanishes in the time-like region of the phase space, which is relevant for the particle production. A finite result emerges when one dresses the propagators by either extending the resummation in the $p h$ channel to two and higher loops and/or by dressing the propagators in the $p p$ channel to all orders. Ignoring the latter resummation, i.e. using the quasiparticle propagators in the two-loop expansion, misses a number of short-range 
collective effects, such as the LPM quenching of the radiation due to multiple scattering. On the other hand, summing only the ladders in the $p p$ channel does not recover the vector current conservation in the radiation process (in, at least, a transparent manner). Therefore a natural choice, motivated by the separation of the short and long range phenomena, is to truncate the $p h$ channel at two-loops and to resum the $p p$ channel to all orders. The situation is reminiscent of the parquet resummation scheme in the first iteration, where in both channels the driving force is the bare baryon-baryon interaction.

Early studies of the bremsstrahlung at the quasiparticle level modelled the strong force using the $T$-matrix interaction [3], the free-space one-boson exchange interaction [4] and their in-medium modifications [5] supplemented with a hard core modelled in the spirit of the Fermi-liquid theory. The explicit use of the tensor interaction turned out to be crucial as there are significant cancellations among different diagrams, and the surviving contribution is due to a non-trivial contraction between the operator structures of the weak and strong interactions (tensor force) [ []]. This motivates our ansatz for the driving force in the particle-hole $(p h)$ channel of nuclear interaction, which includes explicitly the tensor force contribution. We do not attempt, in the present work, to go beyond the one-pion exchange approximation for several reasons, one being that the non-perturbative treatment of the interaction does not change the spin, isospin, and tensor operator structure of the interaction, and important cancellations in the radiation matrix elements will be preserved in a more advanced treatment. We also want to be able to isolate the finite width effects in our comparisons to the earlier work done in the one-pion exchange approximation [⿴囗十.9. The situation is different in the particle-particle $(p p)$ channel, where the short-range correlations have to be treated in a non-perturbative manner by summing up the ladder diagrams to all orders. We do this in the finite-temperature Brueckner theory.

The paper is organized as follows. In Section 2, starting from the Kadanoff-Baym formalism, we derive a single-time transport equation for (anti)-neutrinos with collision integrals driven by (anti)-neutrino coupling to baryons via the polarization tensor of the medium. The polarization tensor is computed in the $2 \mathrm{p}-2 \mathrm{~h}$ approximation in Section 3. The summation of the ladder diagrams in the $p p$ channel within the finite temperature Brueckner theory is described in Section 4. Section 5 evaluates the phase space integrals and neutrino bremsstrahlung emissivities. The numerical results are presented in Section 6. Section 7 summarizes our main results.

\section{NEUTRINO TRANSPORT FORMALISM}

\section{A. Neutrino propagators}

The theory of neutrino radiation can be conveniently formulated in terms of the realtime quantum neutrino transport. Let us start by defining the various time-ordered Greens functions of massless Dirac neutrinos. These can be written in the generic matrix form

$$
i \underline{S}_{12}=i\left(\begin{array}{cc}
S_{12}^{c} & S_{12}^{<} \\
S_{12}^{>} & S_{12}^{a}
\end{array}\right)=\left(\begin{array}{cc}
\left\langle T \psi\left(x_{1}\right) \bar{\psi}\left(x_{2}\right)\right\rangle & -\left\langle\bar{\psi}\left(x_{2}\right) \psi\left(x_{1}\right)\right\rangle \\
\left\langle\psi\left(x_{1}\right) \bar{\psi}\left(x_{2}\right)\right\rangle & \left\langle\tilde{T} \psi\left(x_{1}\right) \bar{\psi}\left(x_{2}\right)\right\rangle
\end{array}\right)=i\left(\begin{array}{cc}
S_{12}^{--} & S_{12}^{-+} \\
S_{12}^{+-} & S_{12}^{++}
\end{array}\right),
$$

where $\psi(x)$ are the neutrino field operators, $\bar{\psi}=\gamma^{0} \psi^{*}, T$ is the chronological time ordering operator, and $\tilde{T}$ is the anti-chronological time ordering operator; the indexes $1=x_{1}, 2=$ 
$x_{2}, \ldots$ collectively denote the space-time and discrete quantum numbers. The neutrino matrix propagator is further assumed to obey the Dyson equation,

$$
\begin{aligned}
\underline{S}\left(x_{1}, x_{2}\right) & =\underline{S}_{0}\left(x_{1}, x_{2}\right)+\underline{S}_{0}\left(x_{1}, x_{3}\right) \underline{\Omega}\left(x_{3}, x_{2}\right) \underline{S}\left(x_{2}, x_{1}\right) \\
& =\underline{S}_{0}\left(x_{1}, x_{2}\right)+\underline{S}\left(x_{1}, x_{3}\right) \underline{\Omega}\left(x_{3}, x_{2}\right) \underline{S}_{0}\left(x_{2}, x_{1}\right),
\end{aligned}
$$

where $S_{0}\left(x_{1}, x_{2}\right)$ is the free neutrino propagator and $S_{0}^{-1}\left(x_{1}, x_{2}\right) S_{0}\left(x_{1}, x_{2}\right)=\sigma_{z} \delta\left(x_{1}-x_{2}\right)$, $\sigma_{z}$ is the third component of the Pauli matrix, $\underline{\Omega}$ is the neutrino proper self-energy and we assume integration (summation) over the repeated variables. The self-energy $\underline{\Omega}$ is a $2 \times 2$ matrix with elements defined on the contour in terms of the Dyson equation. The quasiclassical neutrino transport equation follows from the Dyson equation in the 'conjugate subtracted' form [21,22]:

$$
i \underline{S}\left(x_{1}, x_{2}\right) \not \partial_{x_{2}}-i \not \partial_{x_{1}} \underline{S}\left(x_{1}, x_{2}\right)=\underline{S}\left(x_{1}, x_{3}\right) \underline{\Omega}\left(x_{3}, x_{2}\right) \underline{\sigma_{z}}-\underline{\sigma_{z}} \underline{\Omega}\left(x_{1}, x_{3}\right) \underline{S}\left(x_{3}, x_{2}\right),
$$

Note that the initial correlations are neglected in eq. (四). The set of the four Green's functions above can be supplemented by the retarded and advanced Green's functions which are defined as

$$
i S_{12}^{R}=\theta\left(t_{1}-t_{2}\right)\left\langle\left\{\psi\left(x_{1}\right), \bar{\psi}\left(x_{2}\right)\right\}\right\rangle, \quad i S_{12}^{A}=-\theta\left(t_{2}-t_{1}\right)\left\langle\left\{\psi\left(x_{1}\right), \bar{\psi}\left(x_{2}\right)\right\}\right\rangle,
$$

where $\theta(x)$ is the Heaviside step function on the real-time contour defined as $d \theta(x) / d x=$ $\sigma_{z} \delta(x)$. The retarded and advanced Green's functions obey integral equations in the quasiclassical limit. The relations between the six Green's functions are listed in the Appendix A. The transport equation for the off-diagonal elements of the matrix Green's function reads

$$
\begin{aligned}
& {\left[\partial_{x_{3}}-\Re \mathrm{e} \Omega^{R}\left(x_{1}, x_{3}\right), S^{>,<}\left(x_{3}, x_{2}\right)\right]-\left[\Re \mathrm{e} S^{R}\left(x_{1}, x_{3}\right), \Omega^{>,<}\left(x_{3}, x_{2}\right)\right]} \\
& =\frac{1}{2}\left\{S^{>,<}\left(x_{1}, x_{3}\right), \Omega^{>,<}\left(x_{3}, x_{2}\right)\right\}+\frac{1}{2}\left\{\Omega^{>,<}\left(x_{1}, x_{3}\right), S^{>,<}\left(x_{3}, x_{2}\right)\right\},
\end{aligned}
$$

where $[$,$] and \{$,$\} stand for commutator and anti-commutator, respectively. In arriving at$ eq. (6) we assumed the existence of the Lehmann representation for the neutrino propagators; as a results we have $\Re$ e $S^{R}=\Re$ e $S^{A} \equiv \Re$ e $S$ and $\Re$ e $\Omega^{R}=\Re$ e $\Omega^{A} \equiv \Re$ e $\Omega$.

For the present purposes the neutrino dynamics can be treated semiclassically, by separating the slowly varying center-of-mass coordinates from the rapidly varying relative coordinates. Carrying out a Fourier transform with respect to the relative coordinates and keeping the first-order gradients in the slow variable we arrive at a quasiclassical neutrino transport equation

$$
\begin{aligned}
i\left\{\Re \mathrm{e} S^{-1}(q, x), S^{>,<}(q, x)\right\}_{P . B .}+i\left\{\Re \mathrm{e} S(q, x), \Omega^{>,<}(q, x)\right\}_{P . B .} \\
=S^{>,<}(q, x) \Omega^{>,<}(q, x)+\Omega^{>,<}(q, x) S^{>,<}(q, x),
\end{aligned}
$$

where $q \equiv\left(\boldsymbol{q}, q_{0}\right)$ and $x$ are the neutrino four momentum and the center-of-mass space-time coordinate, respectively, $\{\ldots\}_{P . B}$ is the four-dimensional Poisson bracket. The l.h.s. of eq. (7) is the precursor of the drift term of the Boltzmann equation. The second Poisson bracket, however, does not fit in the Boltzmann description and can be eliminated by an expansion of the neutrino propagator in the leading (quasi-particle) and next-to-leading order terms 
in the small neutrino damping: $S^{>,<}(q, x)=S_{0}^{>,<}(q, x)+S_{1}^{>,<}(q, x)$. A direct evaluation of the Poisson brackets decouples the l.h.s. of transport equation (17) to the leading order with respect to the small damping of neutrino/anti-neutrino states $(\Im \mathrm{m} \Omega(q, x) / \Re \mathrm{e} \Omega(q, x) \ll 1)$. The quasiparticle part of the transport equation

$$
i\left\{\Re \mathrm{e} S^{-1}(q, x), S_{0}^{>,<}(q, x)\right\}_{P . B .}=S^{>,<}(q, x) \Omega^{>,<}(q, x)+\Omega^{>,<}(q, x) S^{>,<}(q, x)
$$

describes the evolution of the distribution function (Wigner function) of on-shell excitations with the l.h.s. corresponding to the drift term of the Boltzmann equation. The r.h.s. corresponds to the collision integral with the self-energies $\Omega^{>},<(q, x)$ having the meaning of the collision rates. The advantage of this form of the (generalized) collision integral is that it admits systematic approximations in terms of the Feynman perturbation theory. The remainder part of the transport equation

$$
i\left\{\Re \mathrm{e} S^{-1}(q, x), S_{1}^{>,<}(q, x)\right\}_{P . B .}+i\left\{\Re \mathrm{e} S(q, x), \Omega^{>,<}(q, x)\right\}_{P . B .}=0,
$$

relates the finite width part of the neutrino propagator to the self-energies in a form of a local functional which depends on the local (anti-)neutrino particle distribution function and their coupling to the matter.

\section{B. On-shell neutrino approximation}

The on-mass-shell neutrino propagator is related to the single-time distribution functions (Wigner functions) of neutrinos and anti-neutrinos, $f_{\nu}(q, x)$ and $f_{\bar{\nu}}(q, x)$, via the ansatz

$$
S_{0}^{<}(q, x)=\frac{i \pi \not q}{\omega_{\nu}(\boldsymbol{q})}\left[\delta\left(q_{0}-\omega_{\nu}(\boldsymbol{q})\right) f_{\nu}(q, x)-\delta\left(q_{0}+\omega_{\nu}(\boldsymbol{q})\right)\left(1-f_{\bar{\nu}}(-q, x)\right)\right],
$$

where $\omega_{\nu}(\boldsymbol{q})=c|q|$ is the on-mass-shell neutrino/anti-neutrino energy. Note that the ansatz includes simultaneously the neutrino particle states and anti-neutrino hole states, which propagate in, say, positive time direction. Similarly, the on-shell propagator

$$
S_{0}^{>}(q, x)=-\frac{i \pi \not q}{\omega_{\nu}(\boldsymbol{q})}\left[\delta\left(q_{0}-\omega_{\nu}(\boldsymbol{q})\right)\left(1-f_{\nu}(q, x)\right)-\delta\left(q_{0}+\omega_{\nu}(\boldsymbol{q})\right) f_{\bar{\nu}}(-q, x)\right],
$$

corresponds to the states propagating in the reversed time direction and, hence, includes the anti-neutrino particle states and neutrino hole states.

To recover the Boltzmann drift term in the on-shell limit, we take the trace on both sides of the transport equation (17) and integrate over the (anti-)neutrino energy $q_{0}$. The

first term on l.h.s. of eq. (7) reduces then to the drift term of the Boltzmann equation. The single time Boltzmann equation (hereafter BE) for neutrinos is obtained after integrating over the positive energy range:

$$
\left[\partial_{t}+\vec{\partial}_{q} \omega_{\nu}(\boldsymbol{q}) \vec{\partial}_{x}\right] f_{\nu}(\boldsymbol{q}, x)=\int_{0}^{\infty} \frac{d q_{0}}{2 \pi} \operatorname{Tr}\left[\Omega^{<}(q, x) S_{0}^{>}(q, x)-\Omega^{>}(q, x) S_{0}^{<}(q, x)\right] ;
$$

a similar equation follows for the anti-neutrinos if one integrates in eq. (7) over the range $[-\infty, 0]$. 


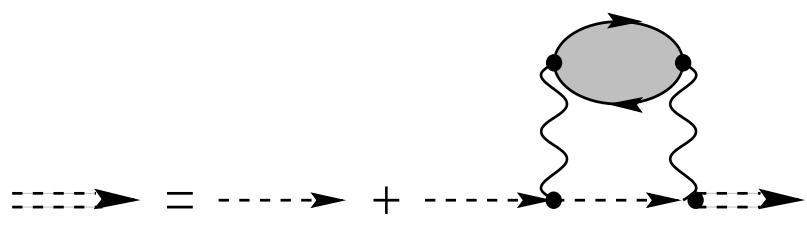

Fig 1: The neutrino Dyson equation in terms of the Feynman diagrams. The dashed curve corresponds to the $S$-propagator, which includes the neutrinos and anti-neutrino holes moving in the same time direction; (reverting the time-direction one finds the Dyson equation for anti-neutrinos and neutrino holes). The shaded loop is the baryon polarization tensor. The wavy lines correspond to the $W^{ \pm}, Z^{0}$ boson propagators.

The different energy integration limits select from the r.h.s. of the transport equations the processes leading to modifications of the distribution functions of (anti-)neutrinos. The separation of the transport equation into neutrino and anti-neutrino parts is arbitrary, however is motivated by the observation that the fundamental quantities of neutrino radiative transport, as the energy densities or neutrino fluxes, can be obtained by taking the appropriate moments of BEs. These quantities are not symmetric with respect to the neutrino/antineutrino populations in general. E.g. the neutrino emissivities (energy output per unit time per unit volume) for processes based on $\beta$-decay reactions are given by the zeroth order moment of the anti-neutrino $\mathrm{BE}$, and it is sufficient to consider only the $\mathrm{BE}$ for anti-neutrinos. In the case of the bremsstrahlung we have to eventually sum these equations; still the relation of the transport self-energies to particular processes becomes transparent if one treats the transport equations separately.

\section{Collision integrals}

We adopt the standard model for the description of the neutrino-baryon interactions and write the neutral current interaction Hamiltonian in the from:

$$
H_{\text {int }}=\frac{G}{2 \sqrt{2}} \Gamma^{H} \Gamma^{L}, \quad \Gamma^{H}=\bar{\phi} \gamma_{\mu}\left(c_{V}-c_{A} \gamma_{5}\right) \phi, \quad \Gamma^{L}=\bar{\psi} \gamma^{\mu}\left(1-\gamma_{5}\right) \psi,
$$

where $G$ is the weak coupling constant, $\psi$ and $\phi$ are the neutrino and baryon field operators, $c_{V}$ and $c_{A}$ are the dimensionless weak neutral-current vector and axial vector coupling constants.

The diagrams contributing to the neutrino emission rates can be arranged in a perturbation expansion with respect to the weak interaction. The lowest order in the weak interaction Feynman diagrams which contribute to scattering, emission, and absorption processes are shown in the Fig. 1. The corresponding transport self-energies are read-off from the diagram

$$
-i \Omega^{>,<}\left(q_{1}, x\right)=\int \frac{d^{4} q}{(2 \pi)^{4}} \frac{d^{4} q_{2}}{(2 \pi)^{4}}(2 \pi)^{4} \delta^{4}\left(q_{1}-q_{2}-q\right) i \Gamma_{L q}^{\mu} i S_{0}^{<}\left(q_{2}, x\right) i \Gamma_{L q}^{\dagger \lambda} i \Pi_{\mu \lambda}^{><}<(q, x),
$$

where $\Pi_{\mu \lambda}^{><}(q)$ are the off-diagonal elements of the matrix of the baryon polarization tensor, $\Gamma_{L q}^{\mu}$ is the weak interaction vertex. The contact interaction (13) can be used for the energymomentum transfers much smaller than the vector boson mass, $q \ll m_{Z}, m_{W}$. Let us first concentrate on the BE for neutrinos. Define the loss and gain terms of the collision integral as: 


$$
I_{\nu}^{>,<}(\boldsymbol{q}, x)=\int_{0}^{\infty} \frac{d q_{0}}{2 \pi} \operatorname{Tr}\left[\Omega^{>,<}(q, x) S_{0}^{>,<}(q, x)\right] .
$$

Substituting the self-energies and the propagators in the collision integrals we find for, e.g., the gain part:

$$
\begin{aligned}
I_{\nu}^{<}\left(\boldsymbol{q}_{1}, x\right) & =-i \int_{0}^{\infty} \frac{d q_{10}}{2 \pi} \operatorname{Tr}\left\{\int _ { - \infty } ^ { \infty } \frac { d ^ { 4 } q } { ( 2 \pi ) ^ { 4 } } \frac { d ^ { 4 } q _ { 2 } } { ( 2 \pi ) ^ { 4 } } ( 2 \pi ) ^ { 4 } \delta ^ { 4 } ( q _ { 1 } - q _ { 2 } - q ) \Gamma _ { L } ^ { \mu } \frac { \pi \not q _ { 2 } } { \omega _ { \nu } ( \boldsymbol { q } _ { 2 } ) } \left[\delta\left(q_{02}-\omega_{\nu}\left(\boldsymbol{q}_{2}\right)\right) f_{\nu}\left(q_{2}, x\right)\right.\right. \\
& \left.\left.-\delta\left(q_{02}+\omega_{\nu}\left(\boldsymbol{q}_{2}\right)\right)\left(1-f_{\bar{\nu}}\left(-q_{2}, x\right)\right)\right] \Gamma_{L}^{\dagger \lambda} \frac{\pi \not q_{1}}{\omega_{\nu}\left(\boldsymbol{q}_{1}\right)} \delta\left(q_{10}-\omega_{\nu}\left(\boldsymbol{q}_{1}\right)\right)\left(1-f_{\nu}\left(q_{1}, x\right)\right) \Pi_{\mu \lambda}^{>}(q, x)\right\} .
\end{aligned}
$$

The loss term is obtained by replacing in eq. (16) the neutrino Wigner functions by the neutrino-hole functions $f_{\nu}(q, x) \rightarrow\left(1-f_{\nu}(q, x)\right)$ and the anti-neutrino-hole Wigner functions by the anti-neutrino functions $\left(1-f_{\bar{\nu}}(-q, x)\right) \rightarrow f_{\bar{\nu}}(q, x)$. The terms proportional $\left(1-f_{\nu}\right) f_{\nu}$ and $\left(1-f_{\nu}\right)\left(1-f_{\bar{\nu}}\right)$ in the gain part of the collision integral, $I_{\nu}^{<}(\boldsymbol{q})$, correspond to the neutrino scattering-in and emission contributions, respectively. The terms proportional $f_{\nu}\left(1-f_{\nu}\right)$ and $f_{\nu} f_{\bar{\nu}}$ in the loss part of the collision integral, $I_{\nu}^{>}(\boldsymbol{q})$, are the neutrino scattering-out and absorption contributions.

The loss and gain collision integrals for the anti-neutrinos can be defined in a manner, similar to the case of neutrinos, with the energy integration spanning the negative energy range

$$
I_{\bar{\nu}}^{>,<}(\boldsymbol{q}, x)=\int_{-\infty}^{0} \frac{d q_{0}}{2 \pi} \operatorname{Tr}\left[\Omega^{>,<}(q, x) S_{0}^{>,<}(q, x)\right] .
$$

Using the above expressions for the self-energy and the propagators, we find, e.g., for the gain term:

$$
\begin{aligned}
I_{\bar{\nu}}^{<}\left(\boldsymbol{q}_{1}, x\right) & =i \int_{-\infty}^{0} \frac{d q_{10}}{2 \pi} \operatorname{Tr}\left\{\int _ { - \infty } ^ { \infty } \frac { d ^ { 4 } q } { ( 2 \pi ) ^ { 4 } } \frac { d ^ { 4 } q _ { 2 } } { ( 2 \pi ) ^ { 4 } } ( 2 \pi ) ^ { 4 } \delta ^ { 4 } ( q _ { 1 } - q _ { 2 } - q ) \Gamma _ { L } ^ { \mu } \frac { \pi \not q _ { 2 } } { \omega _ { \nu } ( \boldsymbol { q } _ { 2 } ) } \left[\delta\left(q_{02}-\omega_{\nu}\left(\boldsymbol{q}_{2}\right)\right) f_{\nu}\left(q_{2}, x\right)\right.\right. \\
& \left.\left.-\delta\left(q_{02}+\omega_{\nu}\left(\boldsymbol{q}_{2}\right)\right)\left(1-f_{\bar{\nu}}\left(-q_{2}, x\right)\right)\right] \Gamma_{L}^{\dagger \lambda} \frac{\pi \not q_{1}}{\omega_{\nu}\left(\boldsymbol{q}_{1}\right)} \delta\left(q_{10}+\omega_{\nu}\left(\boldsymbol{q}_{1}\right)\right) f_{\bar{\nu}}\left(-q_{1}, x\right) \Pi_{\mu \lambda}^{>}(q, x)\right\} .(18)
\end{aligned}
$$

The loss term is obtained by making replacements in eq. (18) analogous to those applied to eq. (16). The terms proportional $f_{\nu} f_{\bar{\nu}}$ and $f_{\bar{\nu}}\left(1-f_{\bar{\nu}}\right)$ in the gain part of the collision integral, $I_{\bar{\nu}}^{<}(\boldsymbol{q})$, then correspond to the neutrino absorption and scattering-out contributions. The terms proportional $\left(1-f_{\bar{\nu}}\right)\left(1-f_{\nu}\right)$ and $\left(1-f_{\bar{\nu}}\right) f_{\bar{\nu}}$ in the loss part of the collision integral, $I_{\bar{\nu}}^{>}(\boldsymbol{q})$, are the neutrino emission and scattering-in contributions, respectively. Note that, when the neutrinos are in a thermal equilibrium with the baryons, the collision integrals for the scattering-in/scattering-out and for the absorption/emission cancel. Under the conditions of detailed balance the (anti-)neutrino distribution function reduces to the Fermi-Dirac form.

\section{Bremsstrahlung emissivity}

The neutrino-pair emissivity (the power of the energy radiated per volume unit) is obtained by multiplying the left-hand-sides of the neutrino and anti-neutrino by their energies, respectively, summing the BEs, and integrating over a phase space element: 


$$
\epsilon_{\nu \bar{\nu}}=\frac{d}{d t} \int \frac{d^{3} q}{(2 \pi)^{3}}\left[f_{\nu}(\boldsymbol{q})+f_{\bar{\nu}}(\boldsymbol{q})\right] \omega_{\nu}(\boldsymbol{q})=\int \frac{d^{3} q}{(2 \pi)^{3}}\left[I_{\nu}^{<, \mathrm{em}}(\boldsymbol{q})-I_{\bar{\nu}}^{>, \mathrm{em}}(\boldsymbol{q})\right] \omega_{\nu}(\boldsymbol{q}),
$$

where in the collision integrals we kept only the terms which correspond to the processes with the neutrino and anti-neutrino in the final state (bremsstrahlung)

$$
\begin{gathered}
\int \frac{d^{3} q_{1}}{(2 \pi)^{3}} I_{\nu}^{>,<, \mathrm{em}}\left(\boldsymbol{q}_{1}\right) \omega_{\nu}\left(\boldsymbol{q}_{1}\right)=i \int \frac{d^{3} q_{1}}{(2 \pi)^{3} 2 \omega_{\nu}\left(\boldsymbol{q}_{1}\right)} \frac{d^{3} q_{2}}{(2 \pi)^{3} 2 \omega_{\nu}\left(\boldsymbol{q}_{2}\right)} \frac{d^{4} q}{(2 \pi)^{4}}(2 \pi)^{4} \delta^{3}\left(\boldsymbol{q}_{1}+\boldsymbol{q}_{2}-\boldsymbol{q}\right) \\
\delta\left(\omega_{\nu}\left(\boldsymbol{q}_{1}\right)+\omega_{\nu}\left(\boldsymbol{q}_{2}\right)-q_{0}\right) \omega_{\nu}\left(\boldsymbol{q}_{1}\right)\left[1-f_{\nu}\left(\omega_{\nu}\left(\boldsymbol{q}_{1}\right)\right)\right]\left[1-f_{\bar{\nu}}\left(\omega_{\nu}\left(\boldsymbol{q}_{2}\right)\right)\right] \Lambda^{\mu \lambda}\left(q_{1}, q_{2}\right) \Pi_{\mu \lambda}^{>><}(q, x),
\end{gathered}
$$

and $\Lambda^{\mu \lambda}=\operatorname{Tr}\left[\gamma^{\mu}\left(1-\gamma^{5}\right) \not \not_{1} \gamma^{\nu}\left(1-\gamma^{5}\right) \not q_{2}\right]$. The collision integrals for neutrinos and antineutrinos can be combined if one uses the identities $\Pi_{\mu \lambda}^{<}(q)=\Pi_{\lambda \mu}^{>}(-q)=2 i g_{B}\left(q_{0}\right) \Im \mathrm{m} \Pi_{\mu \lambda}^{R}(q)$; here $g_{B}\left(q_{0}\right)$ is the Bose distribution function and $\Pi_{\mu \lambda}^{R}(q)$ is the retarded component of the polarization tensor. With these modifications the neutrino-pair bremsstrahlung emissivity becomes

$$
\begin{aligned}
\epsilon_{\nu \bar{\nu}}=-2\left(\frac{G}{2 \sqrt{2}}\right)^{2} \sum_{f} \int \frac{d^{3} q_{2}}{(2 \pi)^{3} 2 \omega_{\nu}\left(\boldsymbol{q}_{2}\right)} \int \frac{d^{3} q_{1}}{(2 \pi)^{3} 2 \omega_{\nu}\left(\boldsymbol{q}_{1}\right)} \int \frac{d^{4} q}{(2 \pi)^{4}} \\
(2 \pi)^{4} \delta^{3}\left(\boldsymbol{q}_{1}+\boldsymbol{q}_{2}-\boldsymbol{q}\right) \delta\left(\omega_{\nu}\left(\boldsymbol{q}_{1}\right)+\omega_{\nu}\left(\boldsymbol{q}_{2}\right)-q_{0}\right)\left[\omega_{\nu}\left(\boldsymbol{q}_{1}\right)+\omega_{\nu}\left(\boldsymbol{q}_{2}\right)\right] \\
g_{B}\left(q_{0}\right)\left[1-f_{\nu}\left(\omega_{\nu}\left(\boldsymbol{q}_{1}\right)\right)\right]\left[1-f_{\bar{\nu}}\left(\omega_{\nu}\left(\boldsymbol{q}_{2}\right)\right)\right] \Lambda^{\mu \lambda}\left(q_{1}, q_{2}\right) \Im \mathrm{m} \Pi_{\mu \lambda}^{R}(q) .
\end{aligned}
$$

We note that eq. (21) is applicable for arbitrary deviation from equilibrium, as the equilibrium properties of the neutrinos and baryons have not been used in the derivation (e.g. the temperature of the bath drops out if one assumes an initially uncorrelated state). Therefore eq. (21) is applicable beyond the boundaries of the linear response theory or the $S$-matrix theory which explicitly resort to the equilibrium properties of the system as a reference point.

\section{TWO-LOOP BARYON POLARIZATION FUNCTION}

In this section we start the implementation of the perturbative scheme motivated in the introduction. Our strategy is the separation of the long and short range phenomena in the $p h$ and $p p$ channels. Here we carry out the first step by expanding the particle-hole channel and truncating it at two loops. This fixes the amount of the long-range correlations in the theory. The short-range effects are condensed in the width of the particle-hole propagators, which is specified in a later section by summing the ladder diagrams.

\section{A. Baryon propagators}

Although we shall treat the baryon sector in the equilibrium limit, it is still useful to define the six Green's functions of the non-equilibrium theory, as in the case of neutrinos. The matrix Green's function of non-relativistic baryons is defined in the standard way

$$
i \underline{G}_{12}=i\left(\begin{array}{cc}
G_{12}^{c} & G_{12}^{<} \\
G_{12}^{>} & G_{12}^{a}
\end{array}\right)=\left(\begin{array}{cc}
\left\langle T \phi\left(x_{1}\right) \phi^{\dagger}\left(x_{2}\right)\right\rangle & -\left\langle\phi^{\dagger}\left(x_{2}\right) \phi\left(x_{1}\right)\right\rangle \\
\left\langle\phi\left(x_{1}\right) \phi^{\dagger}\left(x_{2}\right)\right\rangle & \left\langle\tilde{T} \phi\left(x_{1}\right) \phi^{\dagger}\left(x_{2}\right)\right\rangle
\end{array}\right)=i\left(\begin{array}{cc}
G_{12}^{--} & G_{12}^{-+} \\
G_{12}^{+-} & G_{12}^{++}
\end{array}\right),
$$


where $\phi(x)$ are the baryon field operators. In terms of these operators the retarded and advanced function are defined as

$$
i G_{12}^{R}=\theta\left(t_{1}-t_{2}\right)\left\langle\left\{\phi\left(x_{1}\right), \phi^{\dagger}\left(x_{2}\right)\right\}\right\rangle, \quad i G_{12}^{A}=-\theta\left(t_{2}-t_{1}\right)\left\langle\left\{\phi\left(x_{1}\right), \phi^{\dagger}\left(x_{2}\right)\right\}\right\rangle .
$$

The structure of the proper self-energy matrix $\underline{\Sigma}$ is identical to eq. (22) and its elements are defined via the Dyson equation for baryons:

$$
\begin{aligned}
\underline{G}\left(x_{1}, x_{2}\right) & =\underline{G}_{0}\left(x_{1}, x_{2}\right)+\underline{G}_{0}\left(x_{1}, x_{3}\right) \underline{\Sigma}\left(x_{3}, x_{2}\right) \underline{G}\left(x_{2}, x_{1}\right) \\
& =\underline{G}_{0}\left(x_{1}, x_{2}\right)+\underline{G}\left(x_{1}, x_{3}\right) \underline{\Sigma}\left(x_{3}, x_{2}\right) \underline{G}_{0}\left(x_{2}, x_{1}\right) .
\end{aligned}
$$

In a complete analogy to the neutrino sector, we approximate the Green's functions by their quasiclassical counterparts by defining center-of-mass and relative space-time coordinates and Fourier transform with respect to the relative space-time coordinates. In the equilibrium limit the dependence of the quasiclassical Green's functions on their center-of-mass spacetime coordinate is trivial and can be dropped. The distribution function of the baryons is related to the off-diagonal elements of the matrix Green function by the exact relations

$$
-i G^{<}(p)=a(p) f_{N}(p), \quad i G^{>}(p)=a(p)\left[1-f_{N}(p)\right],
$$

where $a(p)=i\left[G^{R}(p)-G^{A}(p)\right]=i\left[G^{>}(p)-G^{<}(p)\right]$ is the baryon spectral function, $f_{N}(p)=$ $[\exp (\beta(\omega-\mu))+1]^{-1}$ is the Fermi-Dirac distribution function, $\beta=T^{-1}$ is the inverse temperature and $\mu$ is the chemical potential (relations (25) will be refereed to as the Kadanoff-Baym ansatz in the following). The quasiparticle energy, $\varepsilon_{p}=p^{2} / 2 m+\left.\Re \mathrm{e} \Sigma^{R}(p)\right|_{\omega=\varepsilon_{p}}$ follows from the solution of the Dyson equation $G^{R}(p)=\left[\omega-\varepsilon_{p}+i \Im \mathrm{m} \Sigma^{R}(p)\right]^{-1}$. When damping of quasiparticle states is small, $\Im \mathrm{m} \Sigma^{R}(p) \ll \Re \mathrm{e} \Sigma^{R}(p)$, the propagators can be decomposed into quasiparticle and background contributions, e.g.,

$$
G^{<}(p) \simeq 2 \pi i z(\boldsymbol{p}) f_{N}(\boldsymbol{p}) \delta\left(\omega-\varepsilon_{p}\right)-\Sigma^{<}(p) \frac{\mathcal{P}}{\left(\omega-\varepsilon_{p}\right)^{2}}+\mathcal{O}\left(\gamma^{2}\right)
$$

Note that the self-energy appearing in the denominator of the second term of eq. (26) via the dispersion relation is restricted, to the leading order in damping, to the mass-shell. In equilibrium,

$$
i \Sigma^{<}(p)=\gamma(p) f_{N}(p), \quad-i \Sigma^{>}(p)=\gamma(p)\left[1-f_{N}(p)\right]
$$

where $\gamma(p)=-2 \Im \mathrm{m} \Sigma(p)$ is the width of the baryon spectral function. The wave-function renormalization, $z(\boldsymbol{p})$, in the same approximation is

$$
z(\boldsymbol{p})=1-\left.\int \frac{d \omega^{\prime}}{2 \pi} \Im \mathrm{m} \Sigma\left(\omega^{\prime}, \boldsymbol{p}\right) \frac{\mathcal{P}}{\left(\omega^{\prime}-\omega\right)^{2}}\right|_{\omega=\varepsilon_{p}},
$$

where we used the integro-differential form of the Kramers-Kronig relation:

$$
\frac{d}{d \omega} \Re \operatorname{e} \Sigma(\omega, \boldsymbol{p})=\int \frac{d \omega^{\prime}}{\pi} \Im \mathrm{m} \Sigma\left(\omega^{\prime}, \boldsymbol{p}\right) \frac{\mathcal{P}}{\left(\omega-\omega^{\prime}\right)^{2}}
$$


On inserting the expression of the wave-function renormalization (28) in the expansion (26) we find the final form of the propagator

$$
G^{<}(p) \simeq 2 \pi i f_{N}(\boldsymbol{p})-2 \pi i \int \frac{d \omega^{\prime}}{2 \pi} \gamma\left(p^{\prime}\right) \frac{\mathcal{P}}{\left(\omega^{\prime}-\varepsilon_{p}\right)^{2}}\left[\delta\left(\omega-\varepsilon_{p}\right)-\delta\left(\omega-\omega^{\prime}\right)\right] f_{N}(\omega) .
$$

Note that this form of propagator renders the strict fulfillment of the spectral sum rule,

$$
\int \frac{d \omega}{2 \pi} a(p)=1
$$

at any order in the expansion with respect to the damping.

Using the linear relations among the propagators, listed in Appendix A, we find for the causal propagator:

$$
\begin{aligned}
G^{--}(p) & =\frac{\omega-\left(\epsilon_{p}+\Re \mathrm{e} \Sigma(p)-\mu\right)}{\left[\omega-\left(\epsilon_{p}+\Re \mathrm{e} \Sigma(p)-\mu\right)\right]^{2}+[\Im \mathrm{m} \Sigma(p)]^{2}} \\
& -\frac{i \Im \mathrm{m} \Sigma(p)}{\left[\omega-\left(\epsilon_{p}+\Re \mathrm{e} \Sigma(p)-\mu\right)\right]^{2}+[\Im \mathrm{m} \Sigma(p)]^{2}} \tanh \left(\frac{\beta \omega}{2}\right),
\end{aligned}
$$

where $\tanh (\omega / 2) \equiv\left[1-2 f_{N}(\omega)\right]$ and $\epsilon_{p}=p^{2} / 2 m$. As the evaluation of the baryon polarization function requires the causal and acausal Green's functions of the type $G^{--}(q+p)$, we note here that, the denominator of such a function can be expanded in the limit $v q \ll \omega$, where $v \ll 1$ is the characteristic velocity of a baryon,

$$
\left(\omega+\varepsilon_{p}\right)-\varepsilon_{\vec{p}+\vec{q}} \simeq \omega-\boldsymbol{p} \cdot \boldsymbol{q} / m-q \frac{\partial}{\partial p} \Re \mathrm{e} \Sigma(p)-\epsilon_{q} \simeq \omega
$$

to the leading order. The approximation (33) will be referred in the following as the softneutrino approximation. We also employed the non-relativistic limit for baryons. If we use the ansatz $\gamma(-\omega)=\gamma(\omega)$, which is exact in the phenomenological Fermi-liquid theory and will be verified in our microscopic calculations, then

$$
\begin{aligned}
G^{--}( \pm \omega, \boldsymbol{p}) & = \pm \frac{\omega}{\omega^{2}+\gamma(\omega, \boldsymbol{p})^{2} / 4} \mp i \frac{\gamma(\omega, \boldsymbol{p}) / 2}{\omega^{2}+\gamma(\omega, \boldsymbol{p})^{2} / 4} \tanh \left(\frac{\beta \omega}{2}\right) \\
-G^{++}( \pm \omega, \boldsymbol{p}) & = \pm \frac{\omega}{\omega^{2}+\gamma(\omega, \boldsymbol{p})^{2} / 4} \pm i \frac{\gamma(\omega, \boldsymbol{p}) / 2}{\omega^{2}+\gamma(\omega, \boldsymbol{p})^{2} / 4} \tanh \left(\frac{\beta \omega}{2}\right)
\end{aligned}
$$

where the second equation follows from the relation $\left[G^{--}(p)\right]^{*}=-G^{++}(p)$, valid in the momentum representation (see Appendix A). Thus both propagators are odd under the exchange of the sign of $\omega$, a property which will be important in establishing the vector current conservation in the radiation processes discussed below. Since the dependence of the the quasiparticle width on the momentum is weak in the density and temperature range of interest it is useful to define momentum average quasiparticle width which a function only of the frequency. This approximation is implemented in the phase space integrations below. 


\section{B. The interactions}

The central ingredient of a bremsstrahlung process is the modelling of strong the interaction. For the particle-hole interaction a reasonable, but not unique, choice is the one-pion exchange interaction combined with a contact interaction in the spirit of the Fermi-liquid theory:

$$
V_{[p h]}(k)=\left(\frac{f_{\pi}}{m_{\pi}}\right)^{2}\left(\boldsymbol{\sigma}_{1} \cdot \boldsymbol{k}\right) D^{--}(\boldsymbol{k})\left(\boldsymbol{\sigma}_{2} \cdot \boldsymbol{k}\right)+f_{0}+f_{1}(\boldsymbol{\sigma} \cdot \boldsymbol{\sigma}),
$$

where $f_{\pi}$ is the pion decay constant, $m_{\pi}$ is the pion mass, $D^{--}(\boldsymbol{k})$ is the one-shell causal pion propagator, $f_{0}$ and $f_{1}$ are the coupling parameters of the Fermi-liquid theory, $\boldsymbol{\sigma}$ is the vector of the Pauli matrices. The non-relativistic reduction of the neutrino-neutron interaction vertex (13) is

$$
\Gamma_{\mu}^{H}=-\frac{G}{2 \sqrt{2}}\left(\delta_{\mu 0}-g_{A} \delta_{\mu i} \sigma_{i}\right)
$$

where $g_{A}=1.25$ is the axial-vector coupling constant.

\section{Direct contribution to the polarization function}

The three topologically different direct diagrams (i.e. those which do not involve an exchange of outgoing particles) are shown in Fig. 2a-c.
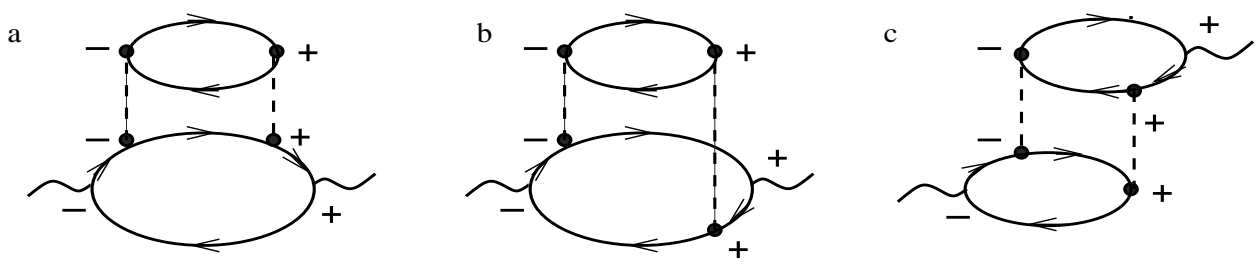

Fig. 2: The Feynman diagrams for neutrino-nucleon interaction in the $2 \mathrm{p}-2 \mathrm{~h}$ approximation. The vertical dashed lines correspond to the baryon-baryon interaction and the wavy lines to the $Z^{0}$ vector bosons. Exchange diagrams are shown below in Fig. 3.

The analytical expression, corresponding to the Fig. 2a, is

$$
\begin{aligned}
i \Pi_{\mu \nu}^{-+, a}(q)= & \int \prod_{i=1}^{4}\left[\frac{d^{4} p_{i}}{(2 \pi)^{4}}\right] \frac{d k}{(2 \pi)^{4}}(2 \pi)^{8} \delta\left(q+p_{4}-k-p_{3}\right) \delta\left(k+p_{2}-p_{1}\right) \operatorname{Tr}\left[V(k) G^{-+}\left(p_{1}\right) V(k) G^{+-}\left(p_{2}\right)\right] \\
& \operatorname{Tr}\left[\Gamma_{\mu} G^{--}\left(q+p_{4}\right) V(k) D^{--}(k) G^{-+}\left(p_{3}\right) V(k) D^{++}(k) G^{++}\left(q+p_{4}\right) \Gamma_{\nu} G^{+-}\left(p_{4}\right)\right],
\end{aligned}
$$

where $V(k)$ is the strong interaction vertex, which can be read-off from eq. (36). The contribution of this diagram is readily recognized as a propagator dressing in the ph channel by means of a self-energy corresponding to an excitation of a single particle-hole collective mode. The analytical expression, corresponding to the Fig. 2b, is 


$$
\begin{aligned}
i \Pi_{\mu \nu}^{-+, b}(q)= & \int \prod_{i=1}^{4}\left[\frac{d^{4} p_{i}}{(2 \pi)^{4}}\right] \frac{d k}{(2 \pi)^{4}}(2 \pi)^{8} \delta\left(q+p_{4}-k-p_{3}\right) \delta\left(k+p_{2}-p_{1}\right) \operatorname{Tr}\left[V(k) G^{-+}\left(p_{1}\right) V(k) G^{+-}\left(p_{2}\right)\right] \\
& \operatorname{Tr}\left[\Gamma_{\mu} G^{--}\left(q+p_{4}\right) V(k) D^{--}(k) G^{-+}\left(p_{3}\right) \Gamma_{\nu} V(k) D^{++}(k) G^{++}\left(p_{3}-q\right) G^{+-}\left(p_{4}\right)\right] .
\end{aligned}
$$

The contribution of this diagram corresponds to a vertex correction in the ph channel by an effective interaction, which incorporates an intermediate particle-hole collective mode excitation. The contribution of the Fig. 2c reads

$$
\begin{aligned}
i \Pi_{\mu \nu}^{-+, c}(q)= & \int \prod_{i=1}^{4}\left[\frac{d^{4} p_{i}}{(2 \pi)^{4}}\right] \frac{d k}{(2 \pi)^{4}}(2 \pi)^{8} \delta\left(q+p_{4}-k-p_{3}\right) \delta\left(k+p_{2}-p_{1}\right) \\
& \operatorname{Tr}\left[\Gamma_{\mu} G^{--}\left(q+p_{4}\right) V(k) D^{--}(k) G^{-+}\left(p_{3}\right) V(k-q) G^{+-}\left(p_{4}\right)\right] \\
& \operatorname{Tr}\left[V(k) G^{-+}\left(p_{1}\right) \Gamma_{\nu} G^{++}\left(p_{1}-q\right) V(k-q) D^{++}(k-q) G^{+-}\left(p_{2}\right)\right] .
\end{aligned}
$$

The latter diagram may be interpreted as a particle-hole fluctuation. The diagrams $a-c$ are evaluated in the Appendix B. There we show that (i) the vector current contributions from diagrams $a$ and $b$ mutually cancel; (ii) the diagram $c$ does not contribute because the axialvector contribution involves traces over odd number of $\sigma$-matrices and the vector-current contribution is cancelled by an equal and of opposite sign contribution from the diagram generated from $c$ by flipping one of the loops upside-down; (iii) all contributions due to the Fermi-liquid interaction cancel after summing the diagrams $a$ and $b$. For the contraction of the trace of the neutrino current with the polarization function we find $(i, j,=1 \ldots 3)$

$$
\begin{aligned}
\mathcal{C}_{\text {dir }}\left(q, \boldsymbol{q}_{1}, \boldsymbol{q}_{2}\right)= & i \operatorname{Tr}\left(\Lambda_{i j}\right)\left[\Pi_{i j}^{-+, a}(q)+\Pi_{i j}^{-+, b}(q)\right] \\
= & 16 g_{A}^{2} G^{2}\left(\frac{f_{\pi}}{m_{\pi}}\right)^{4} \int \prod_{i=1}^{4}\left[\frac{d^{4} p_{i}}{(2 \pi)^{4}}\right] \frac{d^{4} k}{(2 \pi)^{4}} G^{--}(\omega)^{2} D^{--}(k)^{2} \\
& \boldsymbol{k}^{4}\left[\omega_{1} \omega_{2}-\frac{\left(\boldsymbol{q}_{1} \cdot \boldsymbol{k}\right)\left(\boldsymbol{q}_{2} \cdot \boldsymbol{k}\right)}{|k|^{2}}\right] G^{-+}\left(p_{1}\right) G^{+-}\left(p_{2}\right) G^{-+}\left(p_{3}\right) G^{+-}\left(p_{4}\right) \\
& (2 \pi)^{4} \delta\left(q+p_{4}-k-p_{3}\right)(2 \pi)^{4} \delta\left(k+p_{2}-p_{1}\right) .
\end{aligned}
$$

This result is valid in the soft-neutrino and non-relativistic baryon limits. The second term on the r.h.s. in the square bracket can be dropped, as it does not contribute after the phase space integrations. Note that the total number of diagrams of the type $a-c$ is four, if one allows for all possible relabelling of incoming and outgoing (identical) baryons; this forfactor is equal to the symmetry factor by which the total rate must be reduced. We do not include these factors explicitly.

\section{Exchange contribution to the polarization function}

The exchange diagrams are generated from the direct ones by means of interchanging the outgoing propagators in a strong vertex. There is a complete set of diagrams analogous to $a$ and $b$ with exchanged labelling of the hole propagators. These contribute to the contraction 


$$
\begin{array}{r}
\mathcal{C}_{\mathrm{ex}}(q)= \\
16 g_{A}^{2} G^{2}\left(\frac{f_{\pi}}{m_{\pi}}\right)^{4} \omega_{1} \omega_{2} G^{--}(\omega)^{2} \int d k \boldsymbol{k}^{4} D^{--}(\boldsymbol{k})^{2} \\
\int \prod_{i=1}^{4}\left[\frac{d^{4} p_{i}}{(2 \pi)^{4}}\right] G^{-+}\left(p_{1}\right) G^{+-}\left(p_{2}\right) G^{-+}\left(p_{3}\right) G^{+-}\left(p_{4}\right) \\
(2 \pi)^{4} \delta\left(q+k+p_{2}-p_{3}\right) \delta\left(k+p_{4}-p_{1}\right),
\end{array}
$$

in the soft neutrino approximation. The skeleton diagrams which correspond to the interference between the direct and exchange contributions are shown Fig. 3a-d. There are eight diagrams of each type if one allows for all possible relabelling of the propagators.
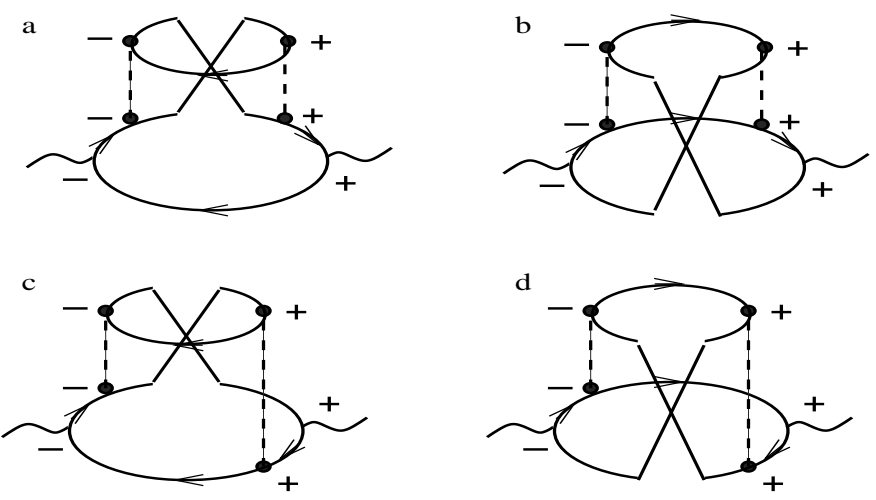

Fig. 3: The exchange Feynman diagrams for baryon-baryon interaction in the $2 \mathrm{p}-2 \mathrm{~h}$ approximation. Conventions are the same as in Fig. 2

The analytical expressions for, e.g., the diagrams $a$ and $c$ are

$$
\begin{aligned}
& i \Pi_{\mu \nu}^{-+, a, \operatorname{ex}}(q)= \int \prod_{i=1}^{4}\left[\frac{d^{4} p_{i}}{(2 \pi)^{4}}\right] \frac{d k}{(2 \pi)^{4}} d k^{\prime}(2 \pi)^{8} \delta\left(q+p_{4}-k-p_{3}\right) \delta\left(k^{\prime}+p_{2}-p_{3}\right) \delta\left(k+p_{2}-p_{1}\right) \\
& \operatorname{Tr}\left[\Gamma_{\mu} G^{--}\left(q+p_{4}\right) V(k) D^{--}(k) G^{-+}\left(p_{3}\right)\right. \\
&\left.V\left(k^{\prime}\right) D^{++}(k) G^{+-}\left(p_{2}\right) V\left(k^{\prime}\right) G^{-+}\left(p_{1}\right) V\left(k^{\prime}\right) G^{++}\left(q+p_{4}\right) \Gamma_{\nu} G^{+-}\left(p_{4}\right)\right], \\
& i \Pi_{\mu \nu}^{-+, c, \operatorname{ex}}(q)= \int \prod_{i=1}^{4}\left[\frac{d^{4} p_{i}}{(2 \pi)^{4}}\right] \frac{d k}{(2 \pi)^{4}} d k^{\prime}(2 \pi)^{8} \delta\left(q+p_{4}-k-p_{3}\right) \delta\left(k^{\prime}+p_{2}-p_{3}\right) \delta\left(k+p_{2}-p_{1}\right) \\
& \operatorname{Tr}\left[\Gamma_{\mu} G^{--}\left(q+p_{4}\right) V(k) D^{--}(k) G^{-+}\left(p_{3}\right)\right. \\
&\left.V\left(k^{\prime}\right) D^{++}(k) G^{+-}\left(p_{2}\right) V\left(k^{\prime}\right) G^{-+}\left(p_{1}\right) \Gamma_{\nu} G^{++}\left(q+p_{4}\right) V\left(k^{\prime}\right) G^{+-}\left(p_{4}\right)\right],
\end{aligned}
$$

and their computation is a complete analogue of that for the direct diagrams (Appendix B). The vector current contribution again cancels among the diagrams $a$ and $c$ and, similarly, $b$ and $d$. The contribution from the interference between the direct and exchange diagrams to the contraction of neutrino and baryon currents is

$$
\begin{aligned}
& \mathcal{C}_{\text {int }}(q)= 16 g_{A}^{2} G^{2}\left(\frac{f_{\pi}}{m_{\pi}}\right)^{4} \omega_{1} \omega_{2} G^{--}(\omega)^{2} \int d k \int d k^{\prime} \boldsymbol{k}^{2} \boldsymbol{k}^{2} D^{--}(\boldsymbol{k}) D^{--}\left(\boldsymbol{k}^{\prime}\right) \\
& \int \prod_{i=1}^{4}\left[\frac{d^{4} p_{i}}{(2 \pi)^{4}}\right] G^{-+}\left(p_{1}\right) G^{+-}\left(p_{2}\right) G^{-+}\left(p_{3}\right) G^{+-}\left(p_{4}\right) \\
&(2 \pi)^{4} \delta\left(q+p_{4}-k-p_{3}\right) \delta\left(k+p_{2}-p_{1}\right) \delta\left(k^{\prime}+p_{4}-p_{1}\right),
\end{aligned}
$$


where we dropped the terms which vanish in the phase space integrations. The phase space integrations in the exchange contribution is complicated, since the momentum integrations do not decouple into two separate loops. The disentanglement can be achieved by constraining the momentum transfer in one of the pion propagators at the value $\left|k^{\prime}\right|=2 p_{F}$, as the main contribution to the integral originates near this value of the momentum transfer.

\section{QUASIPARTICLE WIDTH}

The purpose of this section is to specify the width of baryon propagators. To this end we carry out a full resummation in the particle-particle $(p p)$ channel by solving the scattering $T$-matrix at finite temperatures. Our approach is based on the Brueckner theory with the continuous energy-momentum spectrum of baryons. The non-perturbative treatment of the $p p$ channel is mandatory for including the effects of the short-range correlations due to the repulsive part of the nucleon-nucleon force. These correlations are then responsible for the width of quasiparticle propagators, $\gamma$, in our perturbation expansion in the particle-hole $(p h)$ channel. The $p h$ interactions are dominated by the weaker long-range part of the nucleonnucleon interaction, which makes possible the perturbative treatment of this channel by a truncation at two loops. The contour ordered $T$-matrix in the configuration space is:

$$
\begin{aligned}
& \underline{T}\left(x_{1}, x_{2} ; x_{3}, x_{4}\right)=\underline{V}_{[p p]}\left(x_{1}, x_{2} ; x_{3}, x_{4}\right) \\
& \quad+i \underline{V}_{[p p]}\left(x_{1}, x_{2} ; x_{3}, x_{4}\right) \underline{G}\left(x_{7}, x_{5}\right) \underline{G}\left(x_{8}, x_{6}\right) \underline{T}\left(x_{5}, x_{6} ; x_{3}, x_{4}\right),
\end{aligned}
$$

where $\underline{V}_{[p p]}\left(x_{1}, x_{2} ; x_{3}, x_{4}\right)=\sigma_{z} V_{[p p]}\left(x_{1}, x_{2} ; x_{3}, x_{4}\right)$, is the time-local baryon-baryon interaction in the particle-particle channel. Note that the time locality implies that the $p p$ propagator product $\underline{G} \underline{G} \equiv \underline{G}_{[p p]}$ should be considered as a single matrix. The components of the scattering amplitudes, needed for complete specification of the self-energies, can be chosen as the retarded/advanced ones; the remaining components are provided by the optical theorem. In the quasiclassical limit the retarded/advanced $T$-matrices obey the integral equation

$$
T^{R / A}\left(\boldsymbol{p}, \boldsymbol{p}^{\prime} ; P\right)=V_{[p p]}\left(\boldsymbol{p}, \boldsymbol{p}^{\prime}\right)+i \int \frac{d^{3} p^{\prime \prime}}{(2 \pi)^{3}} V_{[p p]}\left(\boldsymbol{p}, \boldsymbol{p}^{\prime \prime}\right) G_{[p p]}^{R / A}\left(\boldsymbol{p}^{\prime \prime}, P\right) T^{R / A}\left(\boldsymbol{p}^{\prime \prime}, \boldsymbol{p}^{\prime}, P\right),
$$

where we kept the leading order terms in the gradient expansion of the product $G_{[p p]}^{R / A} T^{R / A}$. Here the subscript $[p p]$ indicates the particle-particle channel and $\boldsymbol{p}, P$ are the relative momentum and total four-momentum respectively. The two-particle Green's function, appearing in the kernel of equation (47), is defined as

$$
\begin{aligned}
G_{[p p]}^{R / A}\left(\boldsymbol{p}_{1}, P_{1}\right)=\int \frac{d \omega_{1}}{2 \pi} \int \frac{d^{4} P_{2}}{(2 \pi)^{4}}\left\{G^{>}\left(P_{2} / 2+p_{1}\right) G^{>}\left(P_{2} / 2-p_{1}\right)\right. \\
\left.-G^{<}\left(P_{2} / 2+p_{1}\right) G^{<}\left(P_{2} / 2-p_{1}\right)\right\} \frac{(2 \pi)^{3} \delta^{3}\left(\boldsymbol{P}_{1}-\boldsymbol{P}_{2}\right)}{E_{1}-E_{2} \pm i \delta},
\end{aligned}
$$

where we dropped the irrelevant dependence of the quasiclassical functions on their centerof-mass space-time coordinates. If the particle-hole symmetry is kept in the kernel of the integral equation, the $T$-matrix diverges at the critical temperature of the superfluid phase transition. To be able to apply our computation to the low-temperature regime (and thereby 
avoid the pairing instability in the $T$-matrix) we drop the hole-hole propagators. This is a common approximation in the Brueckner theory and is justified in terms of the BetheGoldstone hole-line expansion. We treat the intermediate state two-particle propagation in the quasiparticle limit. Using the angle averaging procedure for the $p p$ propagator and after partial wave expansion, the thermodynamic retarded $T$-matrix is given by

$$
\begin{aligned}
T_{l l^{\prime}}^{R \alpha}\left(p, p^{\prime}, P, \omega\right) & =V_{[p p] l l^{\prime}}^{\alpha}\left(p, p^{\prime}\right) \\
& +\frac{2}{\pi} \sum_{l^{\prime \prime}} \int d p^{\prime \prime} p^{\prime \prime 2} V_{[p p] l l^{\prime \prime}}^{\alpha}\left(p, p^{\prime \prime}\right)\left\langle G_{[p p]}^{R}\left(p^{\prime \prime}, P, \omega\right)\right\rangle T_{l^{\prime \prime} l^{\prime}}^{R \alpha}\left(p^{\prime \prime}, p^{\prime}, P, \omega\right),
\end{aligned}
$$

where $\alpha$ collectively denotes the quantum numbers $(S, J, M)$ in a particular partial wave, $p$ and $P$ are the magnitudes of the relative and total momentum respectively, $V\left(p, p^{\prime}\right)$ is the bare nuclear interaction. Here $\left\langle G_{[p p]}^{R}\right\rangle$ is the angle averaged two-particle propagator

$$
\left\langle G_{[p p]}^{R}(p, P, \omega)\right\rangle=\int \frac{d \Omega}{4 \pi} \frac{\left[1-f_{N}(\varepsilon(\boldsymbol{P} / 2+\boldsymbol{p}))\right]\left[1-f_{N}(\varepsilon(\boldsymbol{P} / 2-\boldsymbol{p}))\right]}{\omega-\varepsilon(\boldsymbol{P} / 2+\boldsymbol{p})-\varepsilon(\boldsymbol{P} / 2-\boldsymbol{p})+i \delta},
$$

with $\varepsilon(\boldsymbol{p})=\epsilon_{p}+\Re \mathrm{e} \Sigma\left(\varepsilon_{p}, \boldsymbol{p}\right)$, i.e. the intermediate state propagation is treated in the quasiparticle approximation. The retarded self-energy is given by

$$
\Sigma^{R}(p, \omega)=\frac{1}{\pi} \sum_{l \alpha}(2 J+1) \int d p^{\prime} p^{2} T_{l l}^{R \alpha}\left(p, p^{\prime} ; p, p^{\prime} ; \omega+\varepsilon\left(p^{\prime}\right)\right) f_{N}\left(\varepsilon\left(p^{\prime}\right)\right),
$$

which also defines its real and imaginary parts. The coupled equations (49) and (51) are subject to normalization to the total density at a given temperature.

\section{PHASE SPACE INTEGRATIONS}

Let us turn to the task of evaluating the phase space integrals in the expressions for the current contractions. We substitute the Kadanoff-Baym ansatz in eq. (41) and use the identity $f_{N}\left(\varepsilon_{1}\right) f_{N}\left(-\varepsilon_{2}\right)=g\left(\varepsilon_{1}-\varepsilon_{2}\right)\left[f_{N}\left(\varepsilon_{2}\right)-f_{N}\left(\varepsilon_{1}\right)\right]$, which is exact in the equilibrium limit. We then find that the contributions from each loop decouple, i.e.,

$\mathcal{C}_{\text {dir }}(q)=16 g_{A}^{2} G^{2}\left(\frac{f_{\pi}}{m_{\pi}}\right)^{4} \omega_{1} \omega_{2} G^{--}(\omega)^{2} \int \frac{d^{4} k}{(2 \pi)^{4}} \boldsymbol{k}^{4} D^{--}(\boldsymbol{k})^{2} g\left(\omega_{k}\right) g\left(\omega-\omega_{k}\right) L(k) L(q-k)$,

where $\omega_{k}=k_{0}$ and the elementary loop is defined as

$$
L(k)=\int \frac{d^{4} p_{1}}{(2 \pi)^{4}} \frac{d^{4} p_{2}}{(2 \pi)^{4}} a\left(p_{1}\right) a\left(p_{2}\right)\left[f_{N}\left(\varepsilon_{2}\right)-f_{N}\left(\varepsilon_{1}\right)\right](2 \pi)^{4} \delta\left(k+p_{2}-p_{1}\right) .
$$

The exchange contribution $\mathcal{C}_{\text {ex }}$ leads to additional factor of two. The interference contribution decouples only under certain constrains. The single loop, eq. (53), can be evaluated to arbitrary order in the spectral width in general [20]. We shall restrict to the small quasiparticle damping limit and use the the expansion with respect to the width of the spectral function given by eq. (30). 


\section{Leading order}

The lowest order approximation corresponds to the quasiparticle (i.e. zero-width) limit. The contribution from a single loop vanishes in the time-like region of the phase space where $\omega_{k} \geq|\boldsymbol{k}|$. This result is found only if the relativistic kinematics is applied; non-relativistic kinematics leads to spurious terms $\propto m / q$. In the space-like region of the phase space the result is finite. We carry out the energy integrations keeping only the leading order term. Removing one of the trivial momentum delta functions we find

$$
L_{0}(k)=\int \frac{d^{3} p}{(2 \pi)^{3}}\left[f_{N}\left(\varepsilon_{p}\right)-f_{N}\left(\varepsilon_{p+k}\right)\right](2 \pi) \delta\left(\omega_{k}+\varepsilon_{p}-\varepsilon_{p+k}\right) .
$$

The integrations can be carried out exactly

$$
L_{0}(k)=\int \frac{d^{3} p}{(2 \pi)^{3}}\left[f_{N}\left(\varepsilon_{p}\right)-f_{N}\left(\varepsilon_{p+k}\right)\right](2 \pi) \delta\left(\omega_{k}+\varepsilon_{p}-\varepsilon_{p+k}\right)=\frac{m^{* 2}}{2 \pi \beta|k|} \mathcal{L}\left(\omega_{k}, \boldsymbol{k}\right),
$$

where $m^{*}$ is the effective mass of a quasiparticle and

$$
\mathcal{L}\left(\omega_{k}, \boldsymbol{k}\right)=\ln \left|\frac{1+\exp \left[-\beta\left(\varepsilon_{-}(k)-\mu\right)\right]}{1+\exp \left[-\beta\left(\varepsilon_{+}(k)-\mu\right)\right]}\right|,
$$

with $\varepsilon_{ \pm}(k)=\left(\omega_{k}^{2}+\varepsilon_{k}^{2}\right) / 4 \varepsilon_{k} \pm \omega_{k} / 2$. Note that the quasiparticle loop (55) is zero in the time like region $\left(\omega_{k} \geq|\boldsymbol{k}|\right)$, which sets a natural cut-off in the phase space integrations below.

\section{Next-to-leading order}

The next-to-leading order contribution (which is linear in $\gamma$ ) is

$$
\begin{aligned}
L_{1}\left(\omega_{k}, k\right) & =2 \int \frac{d^{4} p}{(2 \pi)^{4}}(2 \pi) \delta\left(\varepsilon+\omega_{k}-\varepsilon_{p+k}\right) \int \frac{d \omega^{\prime}}{2 \pi} \gamma\left(p^{\prime}\right) \frac{\mathcal{P}}{\left(\omega^{\prime}-\varepsilon_{p}\right)^{2}} \\
& \times\left\{\delta\left(\varepsilon-\varepsilon_{p}\right)-\delta\left(\varepsilon-\omega^{\prime}\right)\right\}\left[f_{N}(\varepsilon)-f_{N}\left(\varepsilon+\omega_{k}\right)\right]
\end{aligned}
$$

where we summed the two term arising from the product of the leading and next-to-leading order contribution to $G^{<}(p)$. The angular integral can be carried out analytically to the accuracy $\mathcal{O}\left(\gamma^{2}\right)$. One finds

$$
L_{1}\left(\omega_{k}, k\right)=-\frac{4 m^{* 2}}{k} \int \frac{d \varepsilon_{p}}{(2 \pi)^{2}}\left[f_{N}\left(\varepsilon_{p}\right)-f_{N}\left(\varepsilon_{p}+\omega\right)\right]\left\{\mathcal{Z}\left(\varepsilon_{p}, \boldsymbol{k}\right)-\mathcal{F}\left(\varepsilon_{p}, \boldsymbol{k}, \omega_{k}\right)\right\},
$$

where the first term in the curly brackets is due to the wave-function renormalization

$$
\mathcal{Z}\left(\varepsilon_{p}, \boldsymbol{k}\right)=\theta\left(\varepsilon_{p}-\varepsilon_{\min }\right) \int d \omega \gamma(\omega) \frac{\mathcal{P}}{\left(\omega-\varepsilon_{p}\right)^{2}}, \quad \varepsilon_{\min }=\frac{\left(\omega_{k}-\varepsilon_{q}\right)^{2}}{4 \varepsilon_{q}} .
$$

The second terms is the off-pole contribution and is given by

$$
\mathcal{F}\left(\omega_{k}, \boldsymbol{k}, \varepsilon_{p}\right)=\arctan \left[\frac{\epsilon_{k}-\omega_{k}-\mu+2 \sqrt{\epsilon_{p} \epsilon_{k}}}{\gamma\left(\varepsilon_{p}+\omega_{k}\right) / 2}\right]-\arctan \left[\frac{\epsilon_{k}-\omega_{k}-\mu-2 \sqrt{\epsilon_{p} \epsilon_{k}}}{\gamma\left(\varepsilon_{p}+\omega_{k}\right) / 2}\right] .
$$


The current contraction, which so far includes contributions to all orders in $\gamma$, now can be decomposed in the leading and next-to-leading order terms with respect to $\gamma$, employing the corresponding decomposition for the loops. E.g. for the direct contribution one finds

$$
\begin{aligned}
\mathcal{C}_{\operatorname{dir}}(q)= & 16 g_{A}^{2} G^{2}\left(\frac{f_{\pi}}{m_{\pi}}\right)^{4} \omega_{1} \omega_{2} G^{--}(\omega)^{2} \int \frac{d^{4} k}{(2 \pi)^{4}} \boldsymbol{k}^{4} D^{--}(\boldsymbol{k})^{2} \\
& g\left(\omega_{k}\right) g\left(\omega-\omega_{k}\right)\left[L_{0}(k) L_{0}(q-k)+L_{1}(k) L_{0}(q-k)+L_{0}(k) L_{1}(q-k)\right] .
\end{aligned}
$$

The exchange and interference terms can be decomposed in a similar manner.

\section{Neutrino emissivity}

After the preparatory work above, the computation of the neutrino emissivity is straightforward. We first relate the current contraction to our original expression for the neutrino emissivity by using the relation $-2 g_{B}\left(q_{0}\right) \Im m \Pi_{\mu \nu}^{R}(q)=i \Pi_{\mu \nu}^{<}(q)$. Expression (21) takes the form:

$\epsilon_{\nu \bar{\nu}}=\sum_{f} \int \frac{d^{3} q_{2}}{(2 \pi)^{3} 2 \omega_{\nu}\left(q_{2}\right)} \int \frac{d^{3} q_{1}}{(2 \pi)^{3} 2 \omega_{\nu}\left(q_{1}\right)} \int \frac{d^{4} q}{(2 \pi)^{4}}(2 \pi)^{4} \delta^{4}\left(q_{1}+q_{2}-q\right)\left[\omega_{\nu}\left(\boldsymbol{q}_{1}\right)+\omega_{\nu}\left(\boldsymbol{q}_{2}\right)\right] \mathcal{C}(q)$,

where $\mathcal{C}(q)$ is the sum of the direct, exchange and interference contributions. Let us first compute the contribution from the direct term by substituting eq. (41) for the current contraction. We carry out the integrations over the neutrino phase space and the summation over the three neutrino flavors to find:

$$
\begin{aligned}
\epsilon_{\nu \bar{\nu}}= & \frac{16}{5(2 \pi)^{7}} g_{A}^{2} G_{F}^{2}\left(\frac{f_{\pi}}{m_{\pi}}\right)^{4} \int_{0}^{\infty} d \omega \omega^{6} G^{--}(\omega)^{2} \int d k k^{6} D^{--}(\boldsymbol{k})^{2} \\
& \int d \omega_{k} g\left(\omega_{k}\right) g\left(\omega-\omega_{k}\right)\left[L_{0}(k) L_{0}(q-k)+L_{1}(k) L_{0}(q-k)+L_{0}(k) L_{1}(q-k)\right],
\end{aligned}
$$

where we used $d^{4} k=4 \pi k^{2} d k d \omega_{k}$. Normalizing the energy scales by the temperature and the momenta by $2 p_{F}$ we obtain

$$
\epsilon_{\nu \bar{\nu}}=\frac{32}{5(2 \pi)^{9}} G_{F}^{2} g_{A}^{2}\left(\frac{f_{\pi}}{m_{\pi}}\right)^{4}\left(\frac{m^{*}}{m}\right)^{4} p_{F} I T^{8}=5.5 \times 10^{19} I_{3} T_{9}^{8}\left(\mathrm{erg} \mathrm{cm}^{-3} \mathrm{~s}^{-1}\right)
$$

where $T_{9}$ is the temperature in units of $10^{9} \mathrm{~K}, I_{3}$ is the integral $I$ in units $10^{3}$ defined as

$$
\begin{aligned}
I & =\int_{0}^{\infty} d y y^{6} G^{--}(y)^{2} \mathcal{Q}(y) \int_{0}^{\infty} d x x^{4} D^{--}(x)^{2} \int_{-\infty}^{\infty} d z g(z) g(y-z)\{\mathcal{L}(z, x) \mathcal{L}(y-z, x) \\
& \left.+\frac{2}{\pi} z \mathcal{L}(y-z, x)[\mathcal{F}(z, x)-\mathcal{Z}(z, x)]+\frac{2}{\pi}(y-z) \mathcal{L}(z, x)[\mathcal{F}(y-z, x)-\mathcal{Z}(y-z, x)]\right\} .
\end{aligned}
$$

\footnotetext{
${ }^{1}$ It is understood that the functions of new variables are relabelled.
} 
The explicit dependence of eq. (64) on the temperature and density is the generic one [1 [6]. Additional dependence on these parameters is contained in the integral (65). Note that to avoid spurious contributions from the quasiparticle part, (the first term in curly brackets in (65)), one should restrict the $z$ integration to the space-like region. For the numerical evaluation of the neutrino emissivity we use, following ref. [4], the free space pion propagator:

$$
D^{--}(k)=\left[\boldsymbol{k}^{2}+m_{\pi}^{2}\right]^{-1} .
$$

The free-space approximation should be valid in the vicinity of the nuclear saturation density. The softening of the one-pion exchange (a precursor of the pion-condensation) increases the neutrino emissivity by large factors [5]. We do not attempt to accommodate this effect as our main interest here is the role of the finite width of quasiparticles. The Pauli blocking factor

$$
\mathcal{Q}(y)=30 \int_{0}^{1} d w w^{2}(1-w)^{2}\left[1-f_{\nu}(w y)\right]\left[1-f_{\bar{\nu}}((1-w) y)\right]
$$

accounts for the occupation of neutrino and anti-neutrino final states. In the dilute (anti)neutrino limit $\beta \mu_{\nu_{f}} \ll 1$ (where $\mu_{\nu_{f}}$ is the chemical potential of neutrinos of flavor $f$ ) $\mathcal{Q}(y)=1$.

In the low-temperature limit $\mathcal{L}(z)=z$ and the $z$-integration decouples from the $x$ integration. On imposing $\gamma(\omega) \rightarrow 0$ (quasiparticle limit) one finds that $\mathcal{F}=0$ and $G^{--}(\omega)=$ $\omega^{-2}$. Then the $z$ integration can be carried analytically upon dropping the wave-function renormalization contribution:

$$
\int_{-\infty}^{\infty} d z g(z) g(y-z) z(y-z)=\frac{y\left(y^{2}+4 \pi^{2}\right)}{6\left(e^{y}-1\right)} .
$$

After these manipulations eq. (64) reduces to Friman and Maxwell's result [ref. 四, eq. (47)]. The numerical coefficient in eq. (64), however, is by a factor 3 larger, since Friman and Maxwell do not carry out the summation over the three neutrino flavors at that stage.

The contribution from the exchange current contraction, eq. (42), leads to a factor of 2 in the integral (65). The contribution of the interference term, in the approximation where one of the momentum transfers is fixed at the characteristic value $2 p_{F}$, is

$$
\begin{aligned}
I_{\text {int }} & =\int_{0}^{\infty} d y y^{6} G^{--}(y)^{2} \mathcal{Q}(y) \int_{0}^{\infty} d x x^{2} D^{--}(x) D^{--}(1) \int_{-\infty}^{\infty} d z g(z) g(y-z)\{\mathcal{L}(z, x) \mathcal{L}(y-z, 1) \\
& \left.+\frac{2}{\pi} z \mathcal{L}(y-z, x)[\mathcal{F}(z, 1)-\mathcal{Z}(z, 1)]+\frac{2}{\pi}(y-z) \mathcal{L}(z, 1)[\mathcal{F}(y-z, x)-\mathcal{Z}(y-z, x)]\right\} .
\end{aligned}
$$

\section{RESULTS}

The numerical calculations were carried out for pure neutron matter using the Paris $N N$ interaction keeping $J \leq 4$ partial waves. Fig. 4 displays the real part of the on-shell selfenergy and the half width of the spectral function as a function of the particle momentum for several values of the temperature at the saturation density $n_{s}=0.17 \mathrm{fm}^{-3}$. The width of the 
quasiparticle propagators can be parametrized in terms of the reciprocal of the quasiparticle life time in the Fermi-liquid theory (damping of the zero sound):

$$
\gamma=a T^{2}\left[1+\left(\frac{\omega}{2 \pi T}\right)^{2}\right],
$$

where $a$ is a density dependent phenomenological parameter. The parabolic dependence of the width on the frequency is justified for temperatures below $30 \mathrm{MeV}$ in the range of the densities $n_{s} \leq n \leq 2 n_{s}$. The quadratic dependence of $\gamma$ on the temperature breaks down at slightly lower temperatures. The value of the parameter $a$ weakly depends on the density and is approximately $0.2 \mathrm{MeV}^{-1}$.

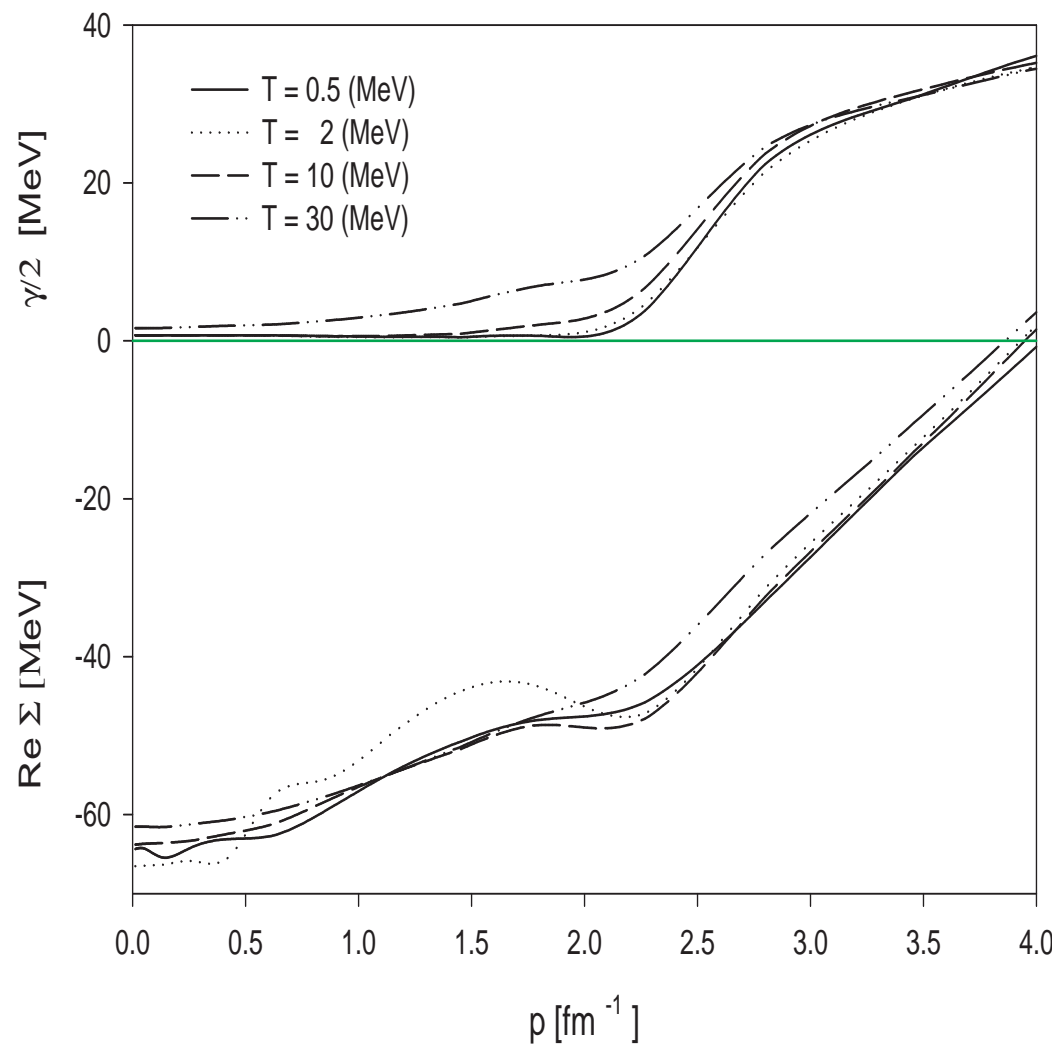

Fig. 4: The real part of the on-shell self-energy and the half-width as a function of particle momentum at the saturation density $n_{s}=0.17 \mathrm{fm}^{-3}$ for different temperatures; the zero temperature Fermi momentum is $1.7 \mathrm{fm}^{-1}$. 
The emergent neutrino spectrum can be caracterized by their spectral function

$$
\begin{aligned}
S(y) & =G^{--}(y)^{2} \mathcal{Q}(y) \int_{0}^{1} d x x^{4} D^{--}(x)^{2} \int_{-\infty}^{\infty} d z g(z) g(y-z)\{\mathcal{L}(z, x) \mathcal{L}(y-z, x) \\
& \left.+\frac{2}{\pi} z \mathcal{L}(y-z, x)[\mathcal{F}(z, x)-\mathcal{Z}(z, x)]+\frac{2}{\pi}(y-z) \mathcal{L}(z, x)[\mathcal{F}(y-z, x)-\mathcal{Z}(y-z, x)]\right\}
\end{aligned}
$$

which is depicted in Fig. 5. The values of the integral are shown as a function of neutrino frequency at $T=20 \mathrm{MeV}$ and the saturation density $n_{s}=0.17 \mathrm{fm}^{-3}$ in the limit of vanishing width (dashed line), including the leading order contribution in the width (dashed-dotted line) and full non-perturbative result (solid line). The energy carried by neutrinos is of order of $\omega \sim 5 T$ in all three cases, as the peak in the spectral function is independent of the approximation to the width of the propagators. The integral $I_{3}$ is show in Fig. 6. The finite width of propagators leads to a suppression of the bremsstrahlung rate as a result of the LPM effect. Keeping the full non-perturbative expression for the causal propagators enhances the value of the integral, as the higher order terms contribute additively to the leading order result. The LPM effect sets in roughly when $\omega \sim \gamma$. As neutrinos are produced thermally, the onset temperature of the LPM effect is of the order of $\gamma$. Equation (69) shows that the value of the parameter a controls the onset temperature which turns out of the order of 5 $\mathrm{MeV}$ in agreement with the previous results of refs. [7-10] and our numerical computation (see Fig. 6).

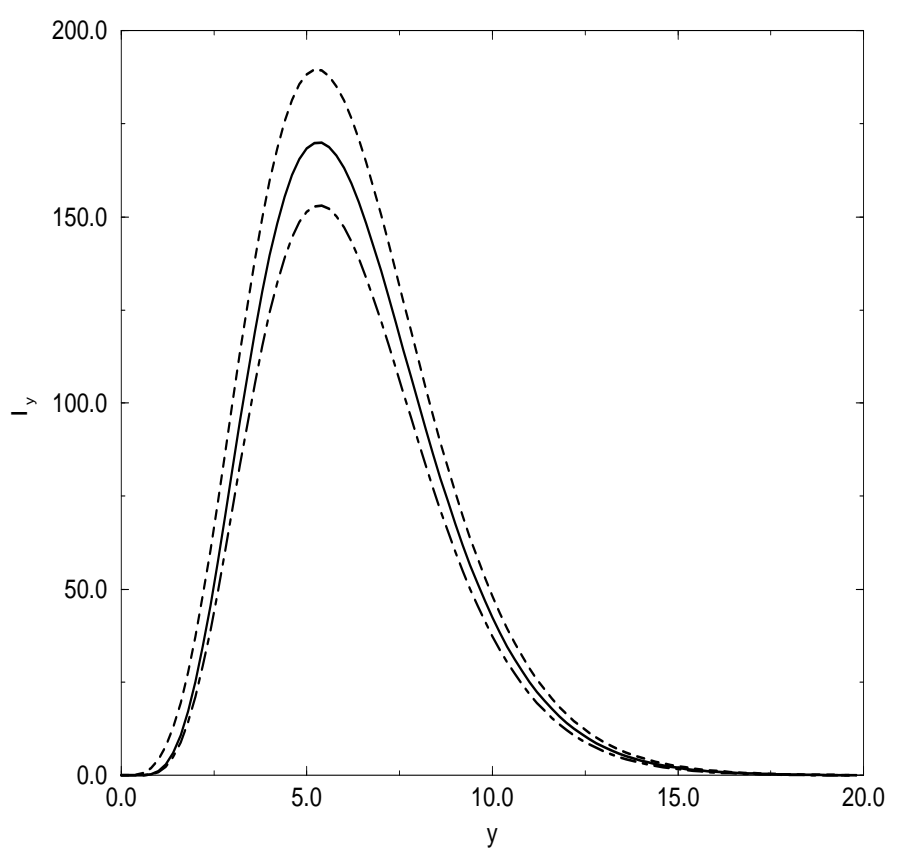

Fig. 5: The neutrino spectral function (70) at the temperature $T=20 \mathrm{MeV}$ and density $n_{s}=0.16$ fm ${ }^{-3}$. The dashed curve is the zero width limit, the dashed-dotted curve includes only the leading order in $\gamma$ contribution from the causal propagator, the solid curve is the full non-perturbative result. 


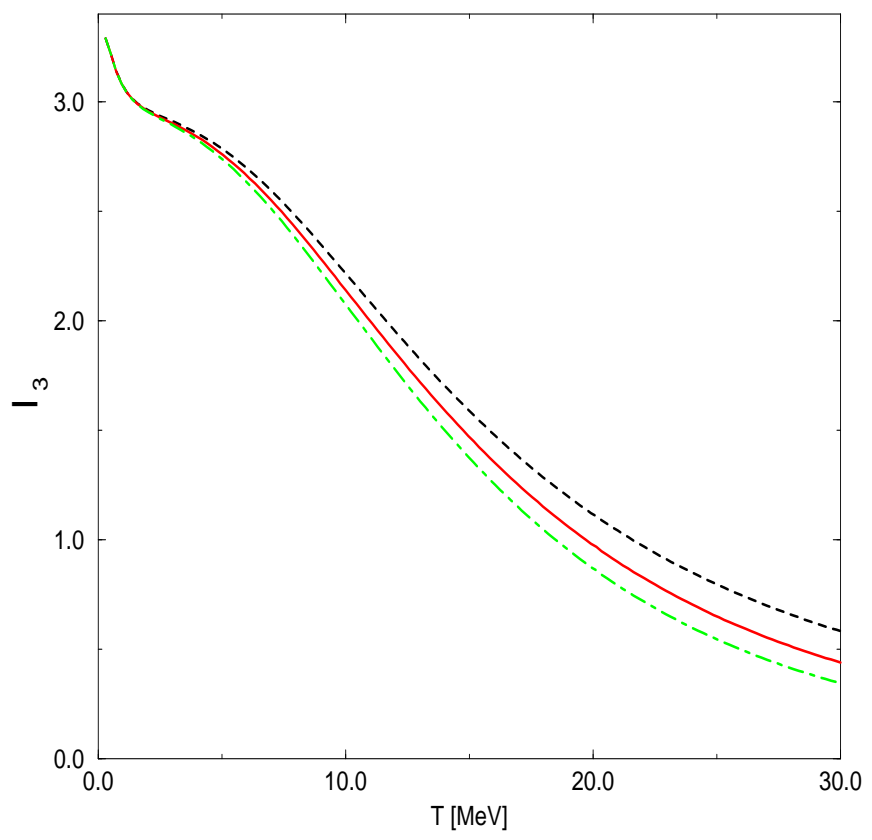

Fig. 6: The integral (65) (including the exchange terms) as a function of temperature at the density $n_{s}=0.16 \mathrm{fm}^{-3}$. The dashed curve is the zero width limit, the dashed-dotted curve includes only the leading order in $\gamma$ contribution from the causal propagator, the solid curve is the full non-perturbative result.

\section{CONCLUSIONS}

In this work we formulated a transport theory for neutrinos in the framework of realtime Green's functions formalism, with particular attention to the collision integrals for the neutrino-pair bremsstrahlung. The main focus was a first principle calculation of the bremsstrahlung emissivity including the width of propagators. This allows to answer questions, not covered by the semi-phenomenological theory, such as the magnitude of the contribution of higher order terms in the expansion with respect to the quasiparticle width or the cancellation of the vector current contribution at all orders in the quasiparticle width. Even though the expression for the emissivity, which follows from our quasiclassical transport equation, is the same as the one found in the linear response theory, it is valid under conditions arbitrary far from equilibrium. This is particularly important in the regime where the neutrinos decouple from matter and their distribution function strongly deviates from the Fermi-Dirac form.

The central quantity of the theory is the particle-hole polarization tensor in the $p h$ channel truncated at two loops. The $p p$ channel is treated non-perturbatively within the finite temperature Brueckner theory. We find that the only contribution to the bremsstrahlung rate comes from the contraction of the tensor force with the axial vector current to all orders in the quasiparticle width. Other contributions, which arise from the contraction of the Fermi-liquid type interaction with the axial vector current and the contraction of the net 
strong interaction with the vector current, cancel when we take the sum of the diagrams corresponding to vertex corrections and propagator renormalization in the $p h$ channel. Thereby the vector current conservation is established at all orders in the quasiparticle width. These cancellations are independent of the approximations to the propagators and are effective both in the quasiparticle limit and beyond. The three ingredients crucial to the cancellations are: (i) the anti-commutation of the tensor force with the axial vector current, (ii) the odd parity of the causal propagator under the exchange of its energy argument, (iii) the soft neutrino and non-relativistic baryon approximations.

Our numerical evaluation of the neutrino emissivity of hot neutron matter, carried out at two loops, shows that the LPM-type suppression sets in at temperatures $T \geq \gamma$, in agreement with the previous work limited to the first order terms in the quasiparticle width (see ref. 9] and references therein). The higher order terms enhance the magnitude of the neutrino emissivity compared to the leading order result. The non-perturbative result, however, is still suppressed as compared to the quasiparticle limit.

Our formalism can be extended in various ways. One obvious extension is allowing for two different chemical potentials of scattering baryons. This will include the Urca process (the $\beta$-decay in the second order in the virial expansion) and the effects of the Pauli spin-paramagnetism, which become important in strong magnetic fields. The formalism can be adapted, with minor changes, for a computation of the space-like analogous of the bremsstrahlung and, in particular, the neutrino opacities of the supernova matter. The perturbative scheme, employed here, itself requires further improvements in several direction, numerically the most important one being the renormalization of the one-boson exchange interaction in the $p h$ channel.

\section{ACKNOWLEDGEMENTS}

This work has been supported by the Stichting voor Fundamenteel Onderzoek der Materie with financial support from the Nederlandse Organisatie voor Wetenschappelijk Onderzoek. A.S. thanks the Institute for Nuclear Theory at the University of Washington for its hospitality and the Department of Energy for partial support during the completion of this work.

\section{APPENDIX A: REAL-TIME GREEN'S FUNCTIONS}

The six Green's functions of the non-equilibrium theory are not independent. For completeness we summarize here the linear relations among them, which can be easily verified from their definitions. The four components of the matrix Green's function are related to each other by the relations

$$
\begin{aligned}
& S^{--}\left(x_{1}, x_{2}\right)=\theta\left(t_{1}-t_{2}\right) S^{+-}\left(x_{1}, x_{2}\right)+\theta\left(t_{2}-t_{1}\right) S^{-+}\left(x_{1}, x_{2}\right), \\
& S^{++}\left(x_{1}, x_{2}\right)=\theta\left(t_{2}-t_{1}\right) S^{+-}\left(x_{1}, x_{2}\right)+\theta\left(t_{1}-t_{2}\right) S^{-+}\left(x_{1}, x_{2}\right), \\
& S^{--}\left(x_{1}, x_{2}\right)+S^{++}\left(x_{1}, x_{2}\right)=S^{-+}\left(x_{1}, x_{2}\right)+S^{+-}\left(x_{1}, x_{2}\right) .
\end{aligned}
$$

Following Hermitian conjugation relations hold: 


$$
\begin{aligned}
& S^{--}\left(x_{1}, x_{2}\right)=-S^{++*}\left(x_{2}, x_{1}\right), \\
& S^{-+}\left(x_{1}, x_{2}\right)=-S^{-+*}\left(x_{2}, x_{1}\right), \\
& S^{--}\left(x_{1}, x_{2}\right)=-S^{+-*}\left(x_{2}, x_{1}\right) .
\end{aligned}
$$

The retarded and advanced Green's functions are related to the components of the matrix Green's function via the relations

$$
\begin{aligned}
S^{R}\left(x_{1}, x_{2}\right) & =\theta\left(t_{1}-t_{2}\right)\left[S^{+-}\left(x_{1}, x_{2}\right)-S^{-+}\left(x_{1}, x_{2}\right)\right] \\
& =S^{--}\left(x_{1}, x_{2}\right)-S^{-+}\left(x_{1}, x_{2}\right)=S^{+-}\left(x_{1}, x_{2}\right)-S^{++}\left(x_{1}, x_{2}\right), \\
S^{A}\left(x_{1}, x_{2}\right) & =-\theta\left(t_{2}-t_{1}\right)\left[S^{+-}\left(x_{1}, x_{2}\right)-S^{-+}(x, y)\right] \\
& =S^{--}\left(x_{1}, x_{2}\right)-S^{+-}\left(x_{1}, x_{2}\right)=S^{-+}\left(x_{1}, x_{2}\right)-S^{++}\left(x_{1}, x_{2}\right) .
\end{aligned}
$$

They are Hermitian conjugates, i.e.

$$
S^{A}\left(x_{1}, x_{2}\right)=S^{R *}\left(x_{1}, x_{2}\right) .
$$

In addition, we note that in the momentum representation they satisfy the equations

$$
S^{--}(\omega, \boldsymbol{p})=-\left[S^{++}(\omega, \boldsymbol{p})\right]^{*}, \quad S^{A}(\omega, \boldsymbol{p})=\left[S^{R}(\omega, \boldsymbol{p})\right]^{*} .
$$

The relations above are valid for the baryon and pion propagators in general, and we do not repeat them here.

Similar relations hold among the self-energies. These can be identified by performing a unitary orthogonal transformation affected by the matrix $R=\left(1+i \sigma_{y}\right) / 2$ by means of formula $S^{\prime}=R^{-1} S R$. The form of the original Dyson equation in the matrix form (3) is invariant against the transformation,

$$
\underline{S^{\prime}}\left(x_{1}, x_{2}\right)=\underline{S}_{0}^{\prime}\left(x_{1}, x_{2}\right)+\underline{S}_{0}^{\prime}\left(x_{1}, x_{3}\right) \underline{\Omega^{\prime}}\left(x_{3}, x_{2}\right) \underline{S^{\prime}}\left(x_{2}, x_{1}\right)
$$

where the primed quantities have the "triangular" form

$$
S_{12}^{\prime}=\left(\begin{array}{cc}
0 & S_{12}^{A} \\
S_{12}^{R} & S_{12}^{K}
\end{array}\right), \quad \Omega_{12}^{\prime}=\left(\begin{array}{cc}
\Omega_{12}^{K} & \Omega_{12}^{R} \\
\Omega_{12}^{A} & 0
\end{array}\right) .
$$

where

$$
\begin{gathered}
S^{K}\left(x_{1}, x_{2}\right)=S^{c}\left(x_{1}, x_{2}\right)+S^{a}\left(x_{1}, x_{2}\right)=S^{>}\left(x_{1}, x_{2}\right)+S^{<}\left(x_{1}, x_{2}\right), \\
\Omega^{R}\left(x_{1}, x_{2}\right)=\Omega^{c}\left(x_{1}, x_{2}\right)+\Omega^{<}\left(x_{1}, x_{2}\right), \quad \Omega^{A}\left(x_{1}, x_{2}\right)=\Omega^{c}\left(x_{1}, x_{2}\right)+\Omega^{>}\left(x_{1}, x_{2}\right), \\
\Omega^{K}\left(x_{1}, x_{2}\right)=\Omega^{c}\left(x_{1}, x_{2}\right)+\Omega^{a}\left(x_{1}, x_{2}\right)=-\Omega^{>}\left(x_{1}, x_{2}\right)-\Omega^{<}\left(x_{1}, x_{2}\right) .
\end{gathered}
$$




\section{APPENDIX B: DETAILS OF THE COMPUTATION OF THE POLARIZATION FUNCTION}

As an example we compute here the direct contribution to the polarization function, represented by the diagrams $a$ and $b$ in Fig. 2. The cancellation among the various contributions from these diagrams does not depend on the details of the structure of the baryon propagators (quasiparticle or dressed), but solely on the odd parity of the causal Green's function with respect to a change of the sign of the energy argument in the soft neutrino approximation.

In the first step we substitute the vertices. As the contribution of the Landau Fermiliquid part of the interaction will cancel out, to save space, we shall drop its contribution from the outset. For the diagrams $a$ and $b$ (excluding the factors for the topologically equivalent diagrams) we find

$$
\begin{aligned}
i \Pi_{\mu \nu}^{-+, a}(q)= & \left(\frac{G}{2 \sqrt{2}}\right)^{2}\left(\frac{f}{m_{\pi}}\right)^{4} \int \prod_{i=1}^{4}\left[\frac{d^{4} p_{i}}{(2 \pi)^{4}}\right] \frac{d k}{(2 \pi)^{4}} \\
& \operatorname{Tr}\left[\left(\delta_{\mu 0}-g_{A} \delta_{\mu i} \sigma_{i}\right) G^{--}\left(q+p_{4}\right)(\boldsymbol{\sigma} \cdot \boldsymbol{k}) D^{--}(k)\right. \\
& \left.G^{-+}\left(p_{3}\right)(\boldsymbol{\sigma} \cdot \boldsymbol{k}) D^{++}(k) G^{++}\left(q+p_{4}\right)\left(\delta_{\nu 0}-g_{A} \delta_{\nu j} \sigma_{j}\right) G^{+-}\left(p_{4}\right)\right] \\
& \operatorname{Tr}\left[(\boldsymbol{\sigma} \cdot \boldsymbol{k}) G^{-+}\left(p_{1}\right)(\boldsymbol{\sigma} \cdot \boldsymbol{k}) G^{+-}\left(p_{2}\right)\right](2 \pi)^{8} \delta\left(q+p_{4}-k-p_{3}\right) \delta\left(k+p_{2}-p_{1}\right), \\
i \Pi_{\mu \nu}^{-+, b}(q)= & \left(\frac{G}{2 \sqrt{2}}\right)^{2}\left(\frac{f}{m_{\pi}}\right)^{4} \int \prod_{i=1}^{4}\left[\frac{d^{4} p_{i}}{(2 \pi)^{4}}\right] \frac{d k}{(2 \pi)^{4}} \\
& \operatorname{Tr}\left[\left(\delta_{\mu 0}-g_{A} \delta_{\mu i} \sigma_{i}\right) G^{--}\left(q+p_{4}\right)(\boldsymbol{\sigma} \cdot \boldsymbol{k}) D^{--}(k)\right. \\
& \left.G^{-+}\left(p_{3}\right)\left(\delta_{\nu 0}-g_{A} \delta_{\nu j} \sigma_{j}\right)(\boldsymbol{\sigma} \cdot \boldsymbol{k}) D^{++}(k) G^{++}\left(p_{3}-q\right) G^{+-}\left(p_{4}\right)\right] \\
& \operatorname{Tr}\left[(\boldsymbol{\sigma} \cdot \boldsymbol{k}) G^{-+}\left(p_{1}\right)(\boldsymbol{\sigma} \cdot \boldsymbol{k}) G^{+-}\left(p_{2}\right)\right](2 \pi)^{8} \delta\left(q+p_{4}-k-p_{3}\right) \delta\left(k+p_{2}-p_{1}\right) .
\end{aligned}
$$

Next we apply the approximation (33) to the causal and acausal Green's functions and fix their momenta at the corresponding Fermi momentum. Combining the diagrams $a$ and $b$, we find

$$
\begin{aligned}
i \Pi_{\mu \nu}^{-+, a}(q)+i \Pi_{\mu \nu}^{-+, b}(q)= & \left(\frac{G}{2 \sqrt{2}}\right)^{2}\left(\frac{f}{m_{\pi}}\right)^{4} \int \prod_{i=1}^{4}\left[\frac{d^{4} p_{i}}{(2 \pi)^{4}}\right] \frac{d k}{(2 \pi)^{4}} \\
& G^{--}(\omega)^{2} D^{--}(k)^{2} G^{-+}\left(p_{1}\right) G^{+-}\left(p_{2}\right) G^{-+}\left(p_{3}\right) G^{+-}\left(p_{4}\right) \\
& \operatorname{Tr}\left\{\left(\delta_{\mu 0}-g_{A} \delta_{\mu i} \sigma_{i}\right)(\boldsymbol{\sigma} \cdot \boldsymbol{k})(\boldsymbol{\sigma} \cdot \boldsymbol{k})\left(\delta_{\nu 0}-g_{A} \delta_{\nu j} \sigma_{j}\right)\right. \\
& \left.-\left(\delta_{\mu 0}-g_{A} \delta_{\mu i} \sigma_{i}\right)(\boldsymbol{\sigma} \cdot \boldsymbol{k})\left(\delta_{\nu 0}-g_{A} \delta_{\nu j} \sigma_{j}\right)(\boldsymbol{\sigma} \cdot \boldsymbol{k})\right\} \\
& \operatorname{Tr}[(\boldsymbol{\sigma} \cdot \boldsymbol{k})(\boldsymbol{\sigma} \cdot \boldsymbol{k})](2 \pi)^{8} \delta\left(q+p_{4}-k-p_{3}\right) \delta\left(k+p_{2}-p_{1}\right),
\end{aligned}
$$

where we used the conjugation relation ( $\mathrm{A10})$. The terms under the trace $\propto \delta_{0 \mu}, \delta_{0 \nu}$ vanish. The $\Pi_{00}$ component of the polarization is hence zero and the vector current is conserved. The remainder simplifies to 


$$
\begin{aligned}
& i \Pi_{i j}^{-+, a}(q)+i \Pi_{i j}^{-+, b}(q)=g_{A}^{2}\left(\frac{G}{2 \sqrt{2}}\right)^{2}\left(\frac{f}{m_{\pi}}\right)^{4} \int \prod_{i=1}^{4}\left[\frac{d^{4} p_{i}}{(2 \pi)^{4}}\right] \frac{d k}{(2 \pi)^{4}} \\
& {\left[G^{--}(\omega)^{2} D^{--}(k)^{2} G^{-+}\left(p_{1}\right) G^{+-}\left(p_{2}\right) G^{-+}\left(p_{3}\right) G^{+-}\left(p_{4}\right)\right.} \\
& \operatorname{Tr}\left[\sigma_{i}(\boldsymbol{\sigma} \cdot \boldsymbol{k})(\boldsymbol{\sigma} \cdot \boldsymbol{k}) \sigma_{j}-\sigma_{i}(\boldsymbol{\sigma} \cdot \boldsymbol{k}) \sigma_{j}(\boldsymbol{\sigma} \cdot \boldsymbol{k})\right] \operatorname{Tr}[(\boldsymbol{\sigma} \cdot \boldsymbol{k})(\boldsymbol{\sigma} \cdot \boldsymbol{k})] \\
& (2 \pi)^{8} \delta\left(q+p_{4}-k-p_{3}\right) \delta\left(k+p_{2}-p_{1}\right) .
\end{aligned}
$$

The computation of the trace using the $\boldsymbol{\sigma}$-algebra gives

$$
\operatorname{Tr}[(\boldsymbol{\sigma} \cdot \boldsymbol{k})(\boldsymbol{\sigma} \cdot \boldsymbol{k})] \operatorname{Tr}\left[\sigma_{i}(\boldsymbol{\sigma} \cdot \boldsymbol{k})(\boldsymbol{\sigma} \cdot \boldsymbol{k}) \sigma_{j}-\sigma_{i}(\boldsymbol{\sigma} \cdot \boldsymbol{k}) \sigma_{j}(\boldsymbol{\sigma} \cdot \boldsymbol{k})\right]=8 k^{2}\left(k^{2} \delta_{i j}-k_{i} k_{j}\right) .
$$

The contraction of the polarization tensor with the trace of neutrino currents, given by

$$
\operatorname{Tr} \Lambda_{i j}=8\left[q_{1 i} q_{2 j}+q_{1 j} q_{2 i}+\left(\omega_{1} \omega_{2}+\boldsymbol{q}_{1} \cdot \boldsymbol{q}_{2}\right) \delta_{i j}+\epsilon_{i n j m} q_{1}^{n} q_{2}^{m}\right] \text {, }
$$

leads to

$$
8 k^{2} \operatorname{Tr} \Lambda_{i j}\left(k^{2} \delta_{i j}-k_{i} k_{j}\right)=128 k^{4}\left[\omega_{1} \omega_{2}-\frac{\left(\boldsymbol{q}_{1} \cdot \boldsymbol{k}\right)\left(\boldsymbol{q}_{1} \cdot \boldsymbol{k}\right)}{k^{2}}\right] .
$$

Combining eqs. (B4) and (B7) we recover eq. (41).

Let us turn to the fluctuation diagram in Fig. 1c. From the original diagram one can generate three additional ones by turning each of the loops upside-down. Let us combine the diagram in Fig. 1c with its counterpart, say $c^{\prime}$, which results from $c$ by turning the upper loop upside-down. The analytical expression corresponding to their sum is

$$
\begin{aligned}
i \Pi_{\mu \nu}^{-+, c}(q)+i \Pi_{\mu \nu}^{-+, c^{\prime}}(q)= & \left(\frac{G}{2 \sqrt{2}}\right)^{2}\left(\frac{f}{m_{\pi}}\right)^{4} \int \prod_{i=1}^{4}\left[\frac{d^{4} p_{i}}{(2 \pi)^{4}}\right] \frac{d k}{(2 \pi)^{4}} \\
& G^{--}(\omega)^{2} D^{--}(k)^{2} G^{-+}\left(p_{1}\right) G^{+-}\left(p_{2}\right) G^{-+}\left(p_{3}\right) G^{+-}\left(p_{4}\right) \\
& \left\{\operatorname{Tr}\left[\left(\delta_{\mu 0}-g_{A} \delta_{\mu i} \sigma_{i}\right)(\boldsymbol{\sigma} \cdot \boldsymbol{k})(\boldsymbol{\sigma} \cdot \boldsymbol{k})\right] \operatorname{Tr}\left[(\boldsymbol{\sigma} \cdot \boldsymbol{k})\left(\delta_{\nu 0}-g_{A} \delta_{\nu j} \sigma_{j}\right)(\boldsymbol{\sigma} \cdot \boldsymbol{k})\right]\right. \\
- & \left.\operatorname{Tr}\left[(\boldsymbol{\sigma} \cdot \boldsymbol{k})\left(\delta_{\mu 0}-g_{A} \delta_{\mu i} \sigma_{i}\right)(\boldsymbol{\sigma} \cdot \boldsymbol{k})\right] \operatorname{Tr}\left[(\boldsymbol{\sigma} \cdot \boldsymbol{k})\left(\delta_{\nu 0}-g_{A} \delta_{\nu j} \sigma_{j}\right)(\boldsymbol{\sigma} \cdot \boldsymbol{k})\right]\right\} \\
& (2 \pi)^{8} \delta\left(q+p_{4}-k-p_{3}\right) \delta\left(k+p_{2}-p_{1}\right),
\end{aligned}
$$

where we dropped $\boldsymbol{q}$ compared with $\boldsymbol{k}$ in the strong interaction vertex. The contribution due to the axial-vector current vanishes because the traces are over odd number of $\boldsymbol{\sigma}$ matrices; the contribution due the vector current cancels as these are identical for diagrams $c$ and $c^{\prime}$ and are of opposite sign. 


\section{REFERENCES}

[1] H. Y. Chiu and E. E. Salpeter, Phys. Rev. Lett. 12, 413 (1964).

[2] J. N. Bahcall and R. A. Wolf, Phys. Rev. Lett. 14, 343 (1965); Phys. Rev. 140, B1452 (1965).

[3] E. G. Flowers, P. G. Sutherland and J. R. Bond, Phys. Rev. D 12, 315 (1975).

[4] B. L. Friman and O. V. Maxwell, Ap. J. 232, 541 (1979).

[5] D. N. Voskresensky and A. V. Senatorov, Sov. Phys. JETP 63, 885 (1986); Sov. J. Nucl. Phys. 45, 411 (1987).

[6] C. J. Pethick, Rev. Mod. Phys. 64, 1133 (1992).

[7] G. Raffelt and D. Seckel, Phys. Rev. Lett. 67, 2605 (1991); Phys. Rev. D 52, 1780 (1995).

[8] H.-T. Janka, W. Keil, G. Raffelt, D. Seckel, Phys. Rev. Lett. 76, 2621 (1996).

[9] G. Raffelt, Stars as Laboratories for Fundamental Physics (Univ. Chicago Press, Chicago, 1996).

[10] S. Hannestad and G. Raffelt, Astrophys. J. 507, 339 (1998).

[11] L. D. Landau and I. Ya. Pomeranchuk, Dokl. Akad. Nauk SSSR 92535 (1953); ibidem 92, 375 (1953). A. B. Migdal, Phys. Rev. 103, 1811 (1956).

[12] J. Knoll and D. N. Voskresensky, Ann. Phys. (NY) 249, 532 (1996).

[13] N. Iwamoto and C. J. Pethick, Phys. Rev. D 25, 313 (1982).

[14] R. F. Sawyer, Phys. Rev. C 40, 865 (1989); Phys. Rev. Lett., 75, 2260 (1995).

[15] C. J. Horowitz and K. Wehrberger, Nucl. Phys. A 531, 665 (1991).

[16] P. Haensel and A. J. Jerzak, Astron. Astrophys. 179, 127 (1987).

[17] A. Burrows and R. F. Sawyer, Phys. Rev. C 58, 554 (1998).

[18] S. Reddy, M. Prakash and J. M. Lattimer, Phys. Rev. D 58, 013009 (1998).

[19] E. Braaten and R. D. Pisarski, Nucl. Phys. B 337, 569 (1990).

[20] A. Sedrakian and A. Dieperink, Phys. Lett B 463, 145 (1999).

[21] L. P. Kadanoff and G. Baym, Quantum Statistical Mechanics (Benjamin, New York, 1962).

[22] W. Botermans and R. Malfliet, Phys. Rep. 198, 115 (1990). 